\title{
A study of symmetric contractions with an application to generalized fractional differential equations
}

\author{
Aftab Hussain ${ }^{1}$, Fahd Jarad ${ }^{3}$ and Erdal Karapinar ${ }^{2,3,4^{*}}$ (1)
}

\section{"Correspondence:}

erdalkarapinar@tdmu.edu.vn; erdalkarapinar@yahoo.com

${ }^{2}$ Division of Applied Mathematics,

Thu Dau Mot University, Binh

Duong Province, Vietnam

${ }^{3}$ Department of Mathematics, Cankaya University, Mimar Sinan

Caddesi, 06790 Ankara, Turkey Full list of author information is available at the end of the article

\begin{abstract}
This article proposes four distinct kinds of symmetric contraction in the framework of complete F-metric spaces. We examine the condition to guarantee the existence and uniqueness of a fixed point for these contractions. As an application, we look for the solutions to fractional boundary value problems involving a generalized fractional derivative known as the fractional derivative with respect to another function.
\end{abstract}

Keywords: Boundary value problem; Generalized fractional derivative; Fixed point; Measure of noncompactness

\section{Introduction}

Among the most interesting and published research topics in the last few decades, we can count the metric fixed-point theory, see, e.g., [1-8], and fractional differential/integral equations, see, e.g., [9-25]. In [20], the authors considered certain fractional and ordinary differential equations and provided solutions by using the metric fixed-point theory techniques. In this paper, we follows the same direction as in [20] and propose solutions for certain fractional differential equations which are based on the new fixed-point theory approaches.

One of the interesting approaches was introduced in [26], where the authors initiated the idea of interpolative-type contractions and established brand new fixed-point results; see also [27-32]. It is worth mentioning that the abstract space structure is as important as the conditions of the contraction in the fixed-point theory. One of the recent exciting results in this direction was introduced by Jleli and Samet [8], who gave a new extension of a metric notion called $\mathcal{F}$-metric space (abbreviated as $\mathcal{F}$-MS).

Let $\mathcal{F}$ be a set of functions $f:(0,+\infty) \rightarrow(-\infty,+\infty)$ such that

$\left(\mathcal{F}_{1}\right) f$ is nondecreasing, that is, for all $0<c<d$, we have $f(c) \leq f(d)$;

$\left(\mathcal{F}_{2}\right)$ for each sequence $\left\{d_{n}\right\} \subset(0,+\infty)$, we have

$$
\lim _{n \rightarrow+\infty} d_{n}=0 \quad \text { if and only if } \lim _{n \rightarrow+\infty} f\left(d_{n}\right)=-\infty
$$

(c) The Author(s) 2021, corrected publication 2021. This article is licensed under a Creative Commons Attribution 4.0 International License, which permits use, sharing, adaptation, distribution and reproduction in any medium or format, as long as you give appropriate credit to the original author(s) and the source, provide a link to the Creative Commons licence, and indicate if changes were made. The images or other third party material in this article are included in the article's Creative Commons licence, unless indicated otherwise in a credit line to the material. If material is not included in the article's Creative Commons licence and your intended use is not permitted by statutory regulation or exceeds the permitted use, you will need to obtain permission directly from the copyright holder. To view a copy of this licence, visit http://creativecommons.org/licenses/by/4.0/. 
Definition 1.1 ([8]) Let $A \neq \emptyset$ with $D: A \times A \rightarrow[0,+\infty)$ be a given mapping. Suppose there exists $(f, \mu) \in \mathcal{F} \times[0,+\infty)$ such that

$\left(D_{1}\right)(w, v) \in A \times A, D(w, v)=0 \Longleftrightarrow w=v$;

$\left(D_{2}\right) D(w, v)=D(v, w)$ for all $(w, v) \in A \times A$;

$\left(D_{3}\right)$ For every $(w, v) \in A \times A$, for each natural number $N \geq 2$, and for every $\left(u_{i}\right)_{i=1}^{N} \subset A$ with $\left(u_{1}, u_{N}\right)=(w, v)$, we have that

$$
D(w, v)>0 \quad \text { implies } f(D(w, v)) \leq f\left(\sum_{i=1}^{N-1} d\left(u_{i}, u_{i+1}\right)\right)+\mu .
$$

Then, $D$ is said to be an $\mathcal{F}$-metric on $A$.

Here, the pair $(A, D)$ is called an $\mathcal{F}$-MS.

A sequence $\left\{w_{n}\right\}$ in $(A, D)$ is $\mathcal{F}$-Cauchy if $\lim _{n, m \rightarrow \infty} D\left(w_{n}, w_{m}\right)=0$. Furthermore, $(A, D)$ is $\mathcal{F}$-complete if every $\mathcal{F}$-Cauchy sequence is $\mathcal{F}$-convergent in $A$.

The following example was stated in [8].

Example 1.2 The set of natural numbers $\mathbb{N}=X$ is an $\mathcal{F}$-MS if we define $D$ by

$$
D(w, v)= \begin{cases}(w-v)^{2}, & \text { if }(w, v) \in[0,3] \times[0,3], \\ |w-v|, & \text { if }(w, v) \notin[0,3] \times[0,3],\end{cases}
$$

for all $(w, v) \in A \times A, f(t)=\ln (t)$ and $\mu=\ln (3)$. Notice that $D$ is not a metric but $(X, D)$ is an $\mathcal{F}$-MS.

Jleli and Samet [8] proposed a simple fixed-point theorem as follows.

Theorem $1.3([8])$ Let $(A, D)$ be an $\mathcal{F}-M S$. Let $g: A \rightarrow A$ be a self mapping. Suppose the following conditions are met:

(i) $(A, D)$ is $\mathcal{F}$-complete;

(ii) there exists a constant $k \in(0,1)$ such that

$$
D(g(w), g(v)) \leq k D(w, v), \quad(w, v) \in A \times A .
$$

Then, $g$ attains a unique fixed point $w^{*} \in A$.

In 2012, Samet et al. introduced a class of $\alpha$-admissible mappings as follows:

Definition 1.4 ([33]) Let $T: A \rightarrow A$ and $\alpha: A \times A \rightarrow[0,+\infty)$. Then $T$ is said to be $\alpha$ admissible if $w, v \in A, \alpha(w, v) \geq 1$ implies that $\alpha(T w, T v) \geq 1$.

Next, Salimi et al. [34] modified the concept of $\alpha$-admissible mappings as follows:

Definition 1.5 ([34]) Let $T: A \rightarrow A$ and $\alpha, \eta: A \times A \rightarrow[0,+\infty)$ two functions. Then $T$ is called an $\alpha$-admissible mapping with respect to $\eta$ if $w, v \in A, \alpha(w, v) \geq \eta(w, v)$ implies that $\alpha(T w, T v) \geq \eta(T w, T v)$. 
Definition 1.6 ([35]) Consider a metric space $(A, d)$, a mapping $T: A \rightarrow A$, and let $\alpha, \eta: A \times A \rightarrow[0, \infty)$ be two functions. Then the mapping $T$ is called an $\alpha-\eta$-continuous mapping in $(A, d)$ whenever given $w \in A$ and a sequence $\left\{w_{n}\right\}$ with

$$
w_{n} \rightarrow w \quad \text { at } \infty, \quad \alpha\left(w_{n}, w_{n+1}\right) \geq \eta\left(w_{n}, w_{n+1}\right), \quad \forall n \in \mathbb{N} \quad \text { implies } \quad T w_{n} \rightarrow T w .
$$

For more details, see, e.g., [36, 37].

A mapping $T: A \rightarrow A$ is called orbitally continuous at $v \in A$ if $\lim _{n \rightarrow \infty} T^{n} w=v$ implies that $\lim _{n \rightarrow \infty} T^{n} w=T v$. A mapping $T$ is orbitally continuous on $A$ if $T$ is orbitally continuous $\forall v \in A$.

\section{Main results}

In this part, we firstly present a new symmetric fractional $\alpha-\eta$-contraction of type I.

Definition 2.1 Let $T: A \rightarrow A$ be a mapping on an $\mathcal{F}$-metric space $(A, D)$ and consider two functions $\alpha, \eta: A \times A \rightarrow[0,+\infty)$. We say that $T$ is a symmetric fractional $\alpha-\eta$-contraction of type I along with constants $\lambda \in[0,1)$ and $\beta, \hat{w}, \gamma \in(0,1)$ if, whenever $\alpha(w, v) \geq \eta(w, v)$, we have

$$
D(T w, T v) \leq \lambda\left(\check{S}_{1}(w, v)\right)
$$

where

$$
\begin{aligned}
\check{S}_{1}(w, v)= & D(w, v) \cdot[D(w, T w)]^{\frac{1}{(\beta-\hat{w})(\beta-\gamma)}} \cdot[D(v, T v)]^{\frac{1}{(\beta-\hat{w})(\beta-\gamma)}} \\
& \cdot[D(w, T w)+D(v, T v)]^{\frac{1}{(\hat{w}-\beta)(\hat{w}-\gamma)}} \\
& \cdot[D(w, T v)+D(v, T w)]^{\frac{1}{(\gamma-\beta)(\gamma-\hat{w})}}
\end{aligned}
$$

for all $w, v \in A \backslash \operatorname{Fix}(T)$.

Example 2.2 Let $A=\{0,1,2,3\}$ be endowed with an $\mathcal{F}$-metric $D$ defined by

$$
D(w, v)= \begin{cases}(w-v)^{2}, & \text { if }(w, v) \in A \times A, \\ |w-v|, & \text { if }(w, v) \notin A \times A .\end{cases}
$$

Consider $f(t)=\ln (t)$ and $\mu=\ln (3)$. Define $T: A \rightarrow A$ by

$$
T 0=0, \quad T 1=1, \quad T 2=T 3=0,
$$

and $\alpha, \eta: A \times A \rightarrow[0,+\infty)$ by

$$
\alpha(w, v)=\left\{\begin{array}{ll}
1, & \text { if } w, v \in A, \\
0, & \text { otherwise, }
\end{array} \quad \eta(w, v)= \begin{cases}\frac{1}{2}, & \text { if } w, v \in A \\
0, & \text { otherwise }\end{cases}\right.
$$


if $w, v \in A$. Clearly, $\alpha(w, v) \geq \eta(w, v)$ and

$$
\begin{aligned}
& D(T 2, T 3)=0 \\
& \leq \lambda\left[D(2,3) \cdot D(2, T 2)^{\frac{1}{(\beta-\hat{w})(\beta-\gamma)}} \cdot D(3, T 3)^{\frac{1}{(\beta-\hat{w})(\beta-\gamma)}}\right. \\
&\left.\cdot(D(2, T 2)+D(3, T 3))^{\frac{1}{(\hat{w}-\beta)(\hat{w}-\gamma)}}(D(2, T 3)+D(3, T 2))^{\frac{1}{(\gamma-\beta)(\gamma-\hat{w})}}\right] \\
&= \lambda\left[1 \cdot D(2,0)^{\frac{1}{(\beta-\hat{w})(\beta-\gamma)}} D(3,0)^{\frac{1}{(\beta-\hat{w})(\beta-\gamma)}}(D(2,0)+D(3,0))^{\frac{1}{(\hat{w}-\beta)(\hat{w}-\gamma)}}\right. \\
& \cdot\left(D(2,0)+D(3,0)^{\frac{1}{(\gamma-\beta)(\gamma-\hat{w})}}\right] \\
&= \lambda\left[(4)^{\frac{1}{(\beta-\hat{w})(\beta-\gamma)}} \cdot(9)^{\frac{1}{(\beta-\hat{w})(\beta-\gamma)}} \cdot(4+9)^{\frac{1}{(\hat{w}-\beta)(\hat{w}-\gamma)}} \cdot(4+9)^{\frac{1}{(\gamma-\beta)(\gamma-\hat{w})}}\right] \\
& \leq \lambda\left[(4)^{\frac{1}{(\beta-\hat{w})(\beta-\gamma)}} \cdot(9)^{\frac{1}{(\beta-\hat{w})(\beta-\gamma)}} \cdot(4 \cdot 9)^{\frac{1}{(\hat{w}-\beta)(\hat{w}-\gamma)}} \cdot(4 \cdot 9)^{\frac{1}{(\gamma-\beta)(\gamma-\hat{w})}}\right] \\
&= \lambda[(4) \cdot(9)]^{\frac{1}{(\beta-\hat{w})(\beta-\gamma)}+\frac{1}{(\hat{w}-\beta)(\hat{w}-\gamma)}}+\frac{1}{(\gamma-\beta)(\gamma-\hat{w})}=\lambda .
\end{aligned}
$$

Clearly, (2.1) holds for all $w, v \in A \backslash \operatorname{Fix}(T)$, if one takes any values of the constants $\lambda \in$ $[0,1), \beta, \hat{w}, \gamma \in(0,1)$. Note that $T$ has two fixed points, which are 0 and 1 .

Now, we state brand new fixed-point theorems for symmetric fractional $\alpha-\eta$-contraction of type I in an $\mathcal{F}$-complete $\mathcal{F}$-MS setting.

Theorem 2.3 Let $(A, D)$ be a complete $\mathcal{F}$-metric space and $T$ be a symmetric fractional $\alpha-\eta$-contraction of type I satisfying the following:

(i) $T$ is a $\alpha$-admissible mapping with respect to $\eta$;

(ii) there exists a $w_{0} \in A$ such that $\alpha\left(w_{0}, T w_{0}\right) \geq \eta\left(w_{0}, T w_{0}\right)$;

(iii) $T$ is $\alpha-\eta$-continuous.

Then, $T$ possesses a fixed point at $A$.

Proof Let $w_{0}$ be in $A$ such that $\alpha\left(w_{0}, T w_{0}\right) \geq \eta\left(w_{0}, T w_{0}\right)$. For $w_{0} \in A$, we build a sequence $\left\{w_{n}\right\}_{n=1}^{\infty}$ in such a way that $w_{1}=T w_{0}, w_{2}=T w_{1}=T^{2} w_{0}$. Proceeding like this, we obtain $w_{n+1}=T w_{n}=T^{n+1} w_{0}$, for every $n \in \mathbb{N}$. Now, since the mapping $T$ is $\alpha$-admissible with respect to $\eta$, we have $\alpha\left(w_{0}, w_{1}\right)=\alpha\left(w_{0}, T w_{0}\right) \geq \eta\left(w_{0}, T w_{0}\right)=\eta\left(w_{0}, w_{1}\right)$. Carrying on this way, we get

$$
\alpha\left(w_{n-1}, w_{n}\right) \geq \eta\left(w_{n-1}, w_{n}\right)=\eta\left(w_{n-1}, T w_{n-1}\right), \quad \text { for all } n \in \mathbb{N} .
$$

If $w_{n+1}=w_{n}$ for some $n \in \mathbb{N}$ then $w_{n}=w^{*}$ is a fixed point of $T$. So, we assume that $w_{n} \neq w_{n+1}$, accompanied by

$$
D\left(T w_{n-1}, T w_{n}\right)=D\left(w_{n}, T w_{n}\right)>0, \quad \text { for all } n \in \mathbb{N} .
$$

As $T$ is a symmetric fractional $\alpha-\eta$-contraction of type $\mathrm{I}$, for $n \in \mathbb{N}$, we have

$$
\begin{aligned}
D\left(w_{n}, w_{n+1}\right) & =D\left(T w_{n-1}, T w_{n}\right) \\
& \leq \lambda\left[D\left(w_{n-1}, w_{n}\right) \cdot D\left(w_{n-1}, T w_{n-1}\right)^{\frac{1}{(\beta-\hat{w})(\beta-\gamma)}}\right.
\end{aligned}
$$




$$
\begin{aligned}
& \cdot D\left(w_{n}, T w_{n}\right)^{\frac{1}{(\beta-\hat{w})(\beta-\gamma)}} \cdot\left[D\left(w_{n-1}, T w_{n-1}\right)+D\left(w_{n}, T w_{n}\right)\right]^{\frac{1}{(\hat{w}-\beta)(\hat{w}-\gamma)}} \\
& \left.\cdot\left[D\left(w_{n-1}, T w_{n}\right)+D\left(w_{n}, T w_{n-1}\right)\right]^{\frac{1}{(\gamma-\beta)(\gamma-\hat{w})}}\right] \\
& =\lambda\left[D\left(w_{n-1}, w_{n}\right) \cdot D\left(w_{n-1}, w_{n}\right)^{\frac{1}{(\beta-\hat{w})(\beta-\gamma)}} \cdot D\left(w_{n}, w_{n+1}\right)^{\frac{1}{(\beta-\hat{w})(\beta-\gamma)}}\right. \\
& \cdot\left[D\left(w_{n-1}, w_{n}\right)+D\left(w_{n}, w_{n+1}\right)\right]^{\frac{1}{(\hat{w}-\beta)(\hat{w}-\gamma)}} \\
& \left.\cdot\left[D\left(w_{n-1}, w_{n+1}\right)+D\left(w_{n}, w_{n}\right)\right]^{\frac{1}{(\gamma-\beta)(\gamma-\hat{w})}}\right] \\
& \leq \lambda\left[D\left(w_{n-1}, w_{n}\right) \cdot D\left(w_{n-1}, w_{n}\right)^{\frac{1}{(\beta-\hat{w})(\beta-\gamma)}} \cdot D\left(w_{n}, w_{n+1}\right)^{\frac{1}{(\beta-\hat{w})(\beta-\gamma)}}\right. \\
& \cdot\left[D\left(w_{n-1}, w_{n}\right)+D\left(w_{n}, w_{n+1}\right)\right]^{\frac{1}{(\hat{w}-\beta)(\hat{w}-\gamma)}} \\
& \left.\cdot\left[D\left(w_{n-1}, w_{n}\right)+D\left(w_{n}, w_{n+1}\right)\right]^{\frac{1}{(\gamma-\beta)(\gamma-\hat{w})}}\right] \\
& =\lambda\left[D\left(w_{n-1}, w_{n}\right) \cdot D\left(w_{n-1}, w_{n}\right)^{\frac{1}{(\beta-\hat{w})(\beta-\gamma)}} \cdot D\left(w_{n}, w_{n+1}\right)^{\frac{1}{(\beta-\hat{w})(\beta-\gamma)}}\right. \\
& \left.\cdot\left[D\left(w_{n-1}, w_{n}\right)+D\left(w_{n}, w_{n+1}\right)\right]^{\frac{1}{(\hat{w}-\beta)(\hat{w}-\gamma)}+\frac{1}{(\gamma-\beta)(\gamma-\hat{w})}}\right] \\
& \leq \lambda\left[D\left(w_{n-1}, w_{n}\right) \cdot D\left(w_{n-1}, w_{n}\right)^{\frac{1}{(\beta-\hat{w})(\beta-\gamma)}} \cdot D\left(w_{n}, w_{n+1}\right)^{\frac{1}{(\beta-\hat{w})(\beta-\gamma)}}\right. \\
& \left.\cdot\left[D\left(w_{n-1}, w_{n}\right) \cdot D\left(w_{n}, w_{n+1}\right)\right]^{\frac{1}{(\hat{w}-\beta)(\hat{w}-\gamma)}+\frac{1}{(\gamma-\beta)(\gamma-\hat{w})}}\right] \\
& =\lambda D\left(w_{n-1}, w_{n}\right)^{1+\frac{1}{(\beta-\hat{w})(\beta-\gamma)}+\frac{1}{(\hat{w}-\beta)(\hat{w}-\gamma)}+\frac{1}{(\gamma-\beta)(\gamma-\hat{w})}} \\
& \cdot D\left(w_{n}, w_{n+1}\right)^{\frac{1}{(\beta-\hat{w})(\beta-\gamma)}+\frac{1}{(\hat{w}-\beta)(\hat{w}-\gamma)}+\frac{1}{(\gamma-\beta)(\gamma-\hat{w})}} \\
& =\lambda D\left(w_{n-1}, w_{n}\right) \text {. }
\end{aligned}
$$

We deduce that

$$
D\left(w_{n}, w_{n+1}\right) \leq \lambda D\left(w_{n-1}, w_{n}\right)
$$

and $\left\{D\left(w_{n-1}, w_{n}\right)\right\}$ is a nonincreasing sequence with nonnegative terms. Thus, there is a nonnegative constant $\varrho$ such that $\lim _{n \rightarrow \infty} D\left(w_{n-1}, w_{n}\right)=\varrho$. Note that $\varrho \geq 0$. From (2.3), we have

$$
D\left(w_{n}, w_{n+1}\right) \leq \lambda D\left(w_{n-1}, w_{n}\right) \leq \lambda^{n} D\left(w_{0}, w_{1}\right)
$$

which provides that

$$
\sum_{i=n}^{m-1} D\left(w_{i}, w_{i+1}\right) \leq \frac{\lambda^{n}}{1-\lambda} D\left(w_{0}, w_{1}\right), \quad m>n .
$$

In the limit we reach

$$
\lim _{n \rightarrow+\infty} \frac{\lambda^{n}}{1-\lambda} D\left(w_{0}, w_{1}\right)=0,
$$

that is, there exists some $N \in \mathbb{N}$ such that

$$
0<\frac{\lambda^{n}}{1-\lambda} D\left(w_{0}, w_{1}\right)<\delta, \quad n \geq N .
$$


Let $\epsilon>0$ be fixed, $(f, \mu) \in \mathcal{F} \times[0, \infty)$, and let $\left(D_{3}\right)$ be satisfied. By $\left(\mathcal{F}_{2}\right)$, there exists $\delta>0$ such that

$$
0<t<\delta \text { implies } f(t)<f(\epsilon)-\mu \text {. }
$$

Hence by $(2.4)$ and $\left(\mathcal{F}_{1}\right)$, we get

$$
f\left(\sum_{i=n}^{m-1} D\left(w_{i}, w_{i+1}\right)\right) \leq f\left(\frac{\lambda^{n}}{1-\lambda} D\left(w_{0}, w_{1}\right)\right)<f(\epsilon)-\mu
$$

where $m, n \in \mathbb{N}$ with $m>n \geq N$ and $D\left(w_{n}, w_{m}\right)>0$. Therefore, by using $\left(D_{3}\right)$ and (2.5), we have

$$
f\left(D\left(w_{m}, w_{n}\right)\right) \leq f\left(\sum_{i=n}^{m-1}\left(D\left(w_{i}, w_{i+1}\right)\right)\right)+\mu<f(\epsilon),
$$

which by $\left(\mathcal{F}_{1}\right)$ implies

$$
D\left(w_{m}, w_{n}\right)<\epsilon, \quad \text { for } m>n \geq N .
$$

Consequently, $\left\{w_{n}\right\}$ is an $\mathcal{F}$-Cauchy sequence. Since $(A, D)$ is an $\mathcal{F}$-complete metric space, there exists an $w^{*} \in A$ such that $w_{n}$ is $\mathcal{F}$-convergent to $w^{*}$, i.e.,

$$
\lim _{n \rightarrow \infty} D\left(w_{n}, w^{*}\right)=0
$$

Now $T$ is $\alpha-\eta$-continuous, $\alpha\left(w_{n-1}, w_{n}\right) \geq \eta\left(w_{n-1}, w_{n}\right)$, for each $n \in \mathbb{N}$, and so $w_{n+1}=T w_{n} \rightarrow$ $T w^{*}$ as $n \rightarrow \infty$, in other words, $w^{*}=T w^{*}$. Now we are going to prove that $w^{*}$ is a fixed point of $T$. We argue by contradiction by supposing that $D\left(T w^{*}, w^{*}\right)>0$. By $\left(D_{3}\right)$, we have

$$
f\left(D\left(T w^{*}, w^{*}\right)\right) \leq f\left(D\left(T w^{*}, T w_{n}\right)+D\left(T w_{n}, w^{*}\right)\right)+\mu, \quad n \in \mathbb{N} .
$$

Using $\left(\mathcal{F}_{1}\right)$ and the contractivity condition gives

$$
\begin{aligned}
f\left(D\left(T w^{*}, w^{*}\right)\right) \leq f & \left.\lambda\left(\begin{array}{c}
D\left(w^{*}, w_{n}\right) \cdot D\left(T w^{*}, w^{*}\right)^{\frac{1}{(\beta-\hat{w})(\beta-\gamma)}} \cdot D\left(w_{n}, T w_{n}\right)^{\frac{1}{(\beta-\hat{w})(\beta-\gamma)}} \\
\cdot\left[D\left(T w^{*}, w^{*}\right)+D\left(w_{n}, T w_{n}\right)\right]^{\frac{1}{(\hat{w}-\beta)(\hat{w}-\gamma)}} \\
\cdot\left[D\left(T w_{n}, w^{*}\right)+D\left(w_{n}, T w^{*}\right)\right]^{\frac{1}{(\gamma-\beta)(\gamma-\hat{w})}}+D\left(w_{n+1}, w^{*}\right)
\end{array}\right)\right) \\
& +\mu,
\end{aligned}
$$

for all $n \in \mathbb{N}$. On the other hand, by using $\left(\mathcal{F}_{2}\right)$ and (2.6), we get

$$
\lim _{n \rightarrow \infty} f\left(\lambda D\left(w^{*}, w_{n}\right)+D\left(w_{n+1}, w^{*}\right)\right)+\mu=-\infty,
$$

which gives a contradiction. Therefore, $D\left(T w^{*}, w^{*}\right)=0$, and hence $w^{*}$ possesses a fixed point of $T$. 
Theorem 2.4 Let $(A, D)$ be an $\mathcal{F}$-complete $\mathcal{F}$-metric space and $T$ be symmetric fractional $\alpha-\eta$-contraction of type I fulfilling the following conditions:

(i) $T$ is a $\alpha$-admissible mapping with respect to $\eta$;

(ii) there exists a $w_{0} \in A$ such that $\alpha\left(w_{0}, T w_{0}\right) \geq \eta\left(w_{0}, T w_{0}\right)$;

(iii) there is a sequence $\left\{w_{n}\right\}$ in $A$ such that $\alpha\left(w_{n}, w_{n+1}\right) \geq \eta\left(w_{n}, w_{n+1}\right)$ with $w_{n} \rightarrow w^{*}$ as $n \rightarrow \infty$ and that $\alpha\left(w_{n}, w^{*}\right) \geq \eta\left(w_{n}, w^{*}\right)$ holds for each $n \in \mathbb{N}$.

Then, $T$ possesses a fixed point in $A$.

Proof Along the lines of the proof of Theorem 2.3, we acquire $\alpha\left(w_{n}, w^{*}\right) \geq \eta\left(w_{n}, w^{*}\right)$ each $n \in \mathbb{N}$. Using $\left(D_{3}\right)$, we have

$$
f\left(D\left(T w^{*}, w^{*}\right)\right) \leq f\left(D\left(T w^{*}, T w_{n}\right)+D\left(w_{n}, w^{*}\right)\right)+\mu .
$$

From $(2.1)$ and $\left(\mathcal{F}_{1}\right)$, we have

$$
\begin{aligned}
& f\left(D\left(T w^{*}, w^{*}\right)\right) \leq f\left(\left(D\left(T w^{*}, T w_{n}\right)\right)+D\left(T w_{n}, w^{*}\right)\right)+\mu \\
& \leq f\left(\lambda\left(\begin{array}{c}
D\left(w^{*}, w_{n}\right) \cdot D\left(T w^{*}, w^{*}\right)^{\frac{1}{(\beta-\hat{w})(\beta-\gamma)}} \cdot D\left(w_{n}, T w_{n}\right)^{\frac{1}{(\beta-\hat{w})(\beta-\gamma)}} \\
\cdot\left[D\left(T w^{*}, w^{*}\right)+D\left(w_{n}, T w_{n}\right)\right]^{\frac{1}{(\hat{w}-\beta)(\hat{w}-\gamma)}} \\
\cdot\left[D\left(T w_{n}, w^{*}\right)+D\left(w_{n}, T w^{*}\right)\right]^{\frac{1}{(\gamma-\beta)(\gamma-\hat{w})}}+D\left(w_{n+1}, w^{*}\right)
\end{array}\right)\right) \\
&+\mu .
\end{aligned}
$$

Employing (2.6) and

$$
\lim _{n \rightarrow \infty} D\left(w_{n}, w^{*}\right)=0, \quad \text { together with } \lim _{n \rightarrow \infty} D\left(w_{n+1}, w^{*}\right)=0,
$$

we obtain

$$
f\left(D\left(w^{*}, T w^{*}\right)\right) \leq f\left(D\left(w^{*}, T w^{*}\right)\right)+\mu .
$$

Making use of $\left(\mathcal{F}_{2}\right)$, we find that

$$
\lim _{n \rightarrow \infty} f\left(D\left(w^{*}, T w^{*}\right)\right)+\mu=-\infty,
$$

which is a contradiction. Therefore $D\left(w^{*}, T w^{*}\right)=0$. In other words, $w^{*}$ is a fixed point of $T$.

Example 2.5 Consider $A=\mathbb{R} \supset \mathbb{N}$ with an $\mathcal{F}$-metric $D: A \times A \rightarrow[0, \infty)$ defined by

$$
D(w, v)= \begin{cases}(w-v)^{2}, & \text { if }(w, v) \in \mathbb{N} \times \mathbb{N}, \\ |w-v|, & \text { if }(w, v) \notin \mathbb{N} \times \mathbb{N},\end{cases}
$$

accompanied by $f(t)=\ln (t)$ and $\mu=\ln (100)$. Define $T: A \rightarrow A$ by

$$
T w= \begin{cases}1-\frac{w}{2}, & \text { if } w \in \mathbb{N}, \\ 0, & \text { if } w \notin \mathbb{N},\end{cases}
$$


and $\alpha, \eta: A \times A \rightarrow[0,+\infty)$ by

$$
\alpha(w, v)=\left\{\begin{array}{ll}
2, & \text { if } w, v \in[0, \infty), \\
0, & \text { otherwise }
\end{array} \quad \eta(w, v)= \begin{cases}1, & \text { if } w, v \in[0, \infty) \\
0, & \text { otherwise }\end{cases}\right.
$$

Case I. If $w=v$ then, clearly, $D(w, v)=0$. Hence all conditions of Theorem 2.3 are satisfied.

Case II. If $w, v$ are in $\mathbb{N}$, but $T w \notin \mathbb{N}, T v \notin \mathbb{N}$, then

$$
D(T w, T v)=D\left(1-\frac{w}{2}, 1-\frac{v}{2}\right)=\left[\frac{1}{2}|w-v|\right] .
$$

Clearly, $T$ is an $\alpha$-admissible mapping with respect to $\eta$, whenever $\alpha(w, v) \geq \eta(w, v)$, so that

$$
D(T w, T v)=\frac{1}{2}|w-v| \leq \lambda\left[\begin{array}{c}
(v-w)^{2} \cdot\left|\frac{3}{2} w-1\right|^{\frac{1}{(\beta-\hat{w})(\beta-\gamma)}} \cdot\left|\frac{3}{2} v-1\right|^{\frac{1}{(\beta-\hat{w})(\beta-\gamma)}} \\
\cdot\left(\left|\frac{3}{2} w-1\right|+\left|\frac{3}{2} v-1\right|\right)^{\frac{1}{(\hat{w}-\beta)(\hat{w}-\gamma)}} \\
\cdot\left(\left|w-\frac{1}{2} v\right|+\left|v-\frac{1}{2} w\right|\right)^{\frac{1}{(\gamma-\beta)(\gamma-\hat{w})}}
\end{array}\right],
$$

by taking constants $\lambda \in[0,1)$, and $\beta, \hat{w}, \gamma \in(0,1)$, for all $w, v \in \mathbb{N} \backslash \operatorname{Fix}(T)$.

Case III. When both $w, v$ are not in $\mathbb{N}$, we obtain

$$
D(T w, T v)=0,
$$

and then, clearly, $T$ is $\alpha$-admissible mapping with respect to $\eta$, whenever $\alpha(w, v) \geq \eta(w, v)$, so that

$$
D(T w, T v)=0 \leq \lambda\left[|w-v| \cdot|w|^{\frac{1}{(\beta-\hat{w})(\beta-\gamma)}} \cdot|v|^{\frac{1}{(\beta-\hat{w})(\beta-\gamma)}} \cdot(|w|+|v|)^{\frac{1}{(\hat{w}-\beta)(\hat{w}-\gamma)}+\frac{1}{(\gamma-\beta)(\gamma-\hat{w})}}\right],
$$

where $\lambda \in[0,1)$, and $\beta, \hat{w}, \gamma \in(0,1)$, for all $w, v \in \mathbb{N} \backslash \operatorname{Fix}(T)$.

Case IV. If one of $w, v$ is in $\mathbb{N}$ and the other is not in $\mathbb{N}$, we obtain

$$
D(T w, T v)=D\left(1-\frac{w}{2}, 0\right)=\left|1-\frac{w}{2}\right| .
$$

Clearly, $T$ is $\alpha$-admissible mapping with respect to $\eta$, whenever $\alpha(w, v) \geq \eta(w, v)$, so that

$$
D(T w, T v)=\left|1-\frac{w}{2}\right| \leq \lambda\left[\begin{array}{c}
|w-v| \cdot\left|\frac{3}{2} w-1\right|^{\frac{1}{(\beta-\hat{w})(\beta-\gamma)}} \cdot|v|^{\frac{1}{(\beta-\hat{w})(\beta-\gamma)}} \\
\cdot\left(\left|\frac{3}{2} w-1\right|+|v|\right)^{\frac{1}{(\hat{w}-\beta)(\hat{w}-\gamma)}} \\
\cdot\left(|w|+\left|v+\frac{1}{2} w-1\right|\right)^{\frac{1}{(\gamma-\beta)(\gamma-\hat{w})}}
\end{array}\right] .
$$


Therefore, all conditions of Theorem 2.3 are satisfied. Hence $T$ is a symmetric fractional $\alpha-\eta$-contraction of type I.

Definition 2.6 Let $(A, D)$ be an $\mathcal{F}$-metric space $(A, D)$ and $\alpha, \eta: A \times A \rightarrow[0,+\infty)$ two functions. Then $\mathcal{F}$ is said to be $\alpha-\eta$-complete on $A$ if and only if every $\mathcal{F}$-Cauchy sequence $\left\{w_{n}\right\}$, satisfying

$$
\alpha\left(w_{n}, w_{n+1}\right) \geq \eta\left(w_{n}, w_{n+1}\right) \quad \text { for each } n \in \mathbb{N},
$$

$\mathcal{F}$-converges in $A$.

Remark 2.7 Theorems 2.3 and 2.4 also hold for an $\alpha-\eta$-complete $\mathcal{F}$-metric space instead of $\mathcal{F}$-complete $\mathcal{F}$-metric space (for details, see [7]).

\section{Symmetric fractional $\alpha-\eta$-contraction of type II}

In this section, a symmetric fractional $\alpha-\eta$-contraction of type II is introduced in the setting of an $\mathcal{F}$-complete $\mathcal{F}$-metric space. Using this notion, we shall provide a fixed point theorem.

Definition 3.1 Consider a self-mapping $T: A \rightarrow A$ on an $\mathcal{F}$-metric space $(A, D)$ and let two functions $\alpha, \eta: A \times A \rightarrow[0,+\infty)$ be given. We say that $T$ is a symmetric fractional $\alpha-\eta$-contraction of type II provided there are constants $\lambda \in[0,1)$ and $\beta, \hat{w}, \gamma \in(0,1)$ such that, whenever $\alpha(w, v) \geq \eta(w, v)$, we have

$$
D(T w, T v) \leq \lambda\left(\check{S}_{2}(w, v)\right)
$$

where

$$
\check{S}_{2}(w, v)=\left\{\begin{array}{c}
D(w, v) \cdot[D(w, T w)]^{\frac{\beta}{(\beta-\hat{w})(\beta-\gamma)}} \cdot[D(v, T v)]^{\frac{\beta}{(\beta-\hat{w}(\beta-\gamma)}} \\
\cdot[D(w, T w)+D(v, T v)]^{\frac{\hat{w}-\beta)(\hat{w}-\gamma)}{\hat{w}}} \cdot[D(w, T v)+D(v, T w)]^{\frac{\gamma}{(\gamma-\beta)(\gamma-\hat{w})}}
\end{array}\right\},
$$

for all $w, v \in A \backslash \operatorname{Fix}(T)$.

Now we show and demonstrate our next theorem.

Theorem 3.2 Let $(A, D)$ be an $\mathcal{F}$-complete $\mathcal{F}$-metric space and $T$ be a symmetric fractional $\alpha-\eta$-contraction of type II fulfilling the following conditions:

(i) $T$ is a $\alpha$-admissible mapping with respect to $\eta$;

(ii) there exists a $w_{0} \in A$ such that $\alpha\left(w_{0}, T w_{0}\right) \geq \eta\left(w_{0}, T w_{0}\right)$;

(iii) $T$ is $\alpha-\eta$-continuous.

Then, $T$ possesses a fixed point in $A$.

Proof Let $w_{0}$ in $A$ be sucht that $\alpha\left(w_{0}, T w_{0}\right) \geq \eta\left(w_{0}, T w_{0}\right)$. For this $w_{0} \in A$, we build a sequence $\left\{w_{n}\right\}_{n=1}^{\infty}$ in such a way that $w_{1}=T w_{0}, w_{2}=T w_{1}=T^{2} w_{0}$. Proceeding this way, $w_{n+1}=T w_{n}=T^{n+1} w_{0}$, for all $n \in \mathbb{N}$. Since the mapping $T$ is $\alpha$-admissible with respect to $\eta$, $\alpha\left(w_{0}, w_{1}\right)=\alpha\left(w_{0}, T w_{0}\right) \geq \eta\left(w_{0}, T w_{0}\right)=\eta\left(w_{0}, w_{1}\right)$. Carrying on in this way, we obtain

$$
\alpha\left(w_{n-1}, w_{n}\right) \geq \eta\left(w_{n-1}, w_{n}\right)=\eta\left(w_{n-1}, T w_{n-1}\right), \quad \text { for all } n \in \mathbb{N} .
$$


If $w_{n+1}=w_{n}$ for some $n \in \mathbb{N}$ then $w_{n}=w^{*}$ is a fixed point of $T$. So, we assume that $w_{n} \neq w_{n+1}$ and that

$$
D\left(T w_{n-1}, T w_{n}\right)=D\left(w_{n}, T w_{n}\right)>0, \quad \text { for every } n \in \mathbb{N} .
$$

As $T$ is a symmetric fractional $\alpha-\eta$-contraction of type II, for each $n \in \mathbb{N}$, we get

$$
\begin{aligned}
& D\left(w_{n}, w_{n+1}\right)=D\left(T w_{n-1}, T w_{n}\right) \\
& \leq \lambda\left[D\left(w_{n-1}, w_{n}\right) \cdot D\left(w_{n-1}, T w_{n-1}\right)^{\frac{\beta}{(\beta-\hat{w})(\beta-\gamma)}}\right. \\
& \cdot D\left(w_{n}, T w_{n}\right)^{\frac{\beta}{(\beta-\hat{w})(\beta-\gamma)}} \cdot\left[D\left(w_{n-1}, T w_{n-1}\right)+D\left(w_{n}, T w_{n}\right)\right]^{\frac{\hat{\hat{w}}}{(\hat{w}-\beta)(\hat{w}-\gamma)}} \\
& \left.\cdot\left[D\left(w_{n-1}, T w_{n}\right)+D\left(w_{n}, T w_{n-1}\right)\right]^{\frac{\gamma}{(\gamma-\beta)(\gamma-\hat{w})}}\right] \\
& =\lambda\left[D\left(w_{n-1}, w_{n}\right) \cdot D\left(w_{n-1}, w_{n}\right)^{\frac{\beta}{(\beta-\hat{w})(\beta-\gamma)}} \cdot D\left(w_{n}, w_{n+1}\right)^{\frac{\beta}{(\beta-\hat{\psi})(\beta-\gamma)}}\right. \\
& \cdot\left[D\left(w_{n-1}, w_{n}\right)+D\left(w_{n}, w_{n+1}\right)\right]^{\frac{\hat{\hat{w}}}{(\hat{w}-\beta)(\hat{w}-\gamma)}} \\
& \left.\cdot\left[D\left(w_{n-1}, w_{n+1}\right)+D\left(w_{n}, w_{n}\right)\right]^{\frac{\gamma}{(\gamma-\beta)(\gamma-\hat{w})}}\right] \\
& \leq \lambda\left[D\left(w_{n-1}, w_{n}\right) \cdot D\left(w_{n-1}, w_{n}\right)^{\frac{\beta}{(\beta-\hat{w})(\beta-\gamma)}} \cdot D\left(w_{n}, w_{n+1}\right)^{\frac{\beta}{(\beta-\hat{w})(\beta-\gamma)}}\right. \\
& \cdot\left[D\left(w_{n-1}, w_{n}\right)+D\left(w_{n}, w_{n+1}\right)\right]^{\frac{\hat{\hat{w}}}{(\hat{w}-\beta)(\hat{w}-\gamma)}} \\
& \left.\cdot\left[D\left(w_{n-1}, w_{n}\right)+D\left(w_{n}, w_{n+1}\right)\right]^{\frac{\gamma}{(\gamma-\beta)(\gamma-\hat{w})}}\right] \\
& =\lambda\left[D\left(w_{n-1}, w_{n}\right) \cdot D\left(w_{n-1}, w_{n}\right)^{\frac{\beta}{(\beta-\hat{w})(\beta-\gamma)}} \cdot D\left(w_{n}, w_{n+1}\right)^{\frac{\beta}{(\beta-\hat{\psi})(\beta-\gamma)}}\right. \\
& \left.\cdot\left[D\left(w_{n-1}, w_{n}\right)+D\left(w_{n}, w_{n+1}\right)\right]^{\frac{\hat{\hat{w}}}{(\hat{w}-\beta)(\hat{w}-\gamma)}+\frac{\gamma}{(\gamma-\beta)(\gamma-\hat{w})}}\right] \\
& \leq \lambda\left[D\left(w_{n-1}, w_{n}\right) \cdot D\left(w_{n-1}, w_{n}\right)^{\frac{\beta}{(\beta-\hat{w})(\beta-\gamma)}} \cdot D\left(w_{n}, w_{n+1}\right)^{\frac{\beta}{(\beta-\hat{w})(\beta-\gamma)}}\right. \\
& \left.\cdot\left[D\left(w_{n-1}, w_{n}\right) \cdot D\left(w_{n}, w_{n+1}\right)\right]^{\frac{\hat{\hat{w}}-\beta)}{(\hat{w}-\gamma)}+\frac{\gamma}{(\gamma-\beta)(\gamma-\hat{w})}}\right] \\
& =\lambda\left[D\left(w_{n-1}, w_{n}\right)^{1+\frac{\beta}{(\beta-\hat{w})(\beta-\gamma)}+\frac{\hat{w}}{(\hat{w}-\beta)(\hat{w}-\gamma)}+\frac{\gamma}{(\gamma-\beta)(\gamma-\hat{w})}}\right. \\
& \cdot D\left(w_{n}, w_{n+1}\right)^{\frac{\beta}{(\beta-\hat{w})(\beta-\gamma)}+\frac{\hat{w}}{(\hat{w}-\beta)(\hat{w}-\gamma)}+\frac{\gamma}{(\gamma-\beta)(\gamma-\hat{w})}} \\
& =\lambda D\left(w_{n-1}, w_{n}\right)
\end{aligned}
$$

and deduce

$$
D\left(w_{n}, w_{n+1}\right) \leq \lambda D\left(w_{n-1}, w_{n}\right) .
$$

We conclude that $\left\{D\left(w_{n-1}, w_{n}\right)\right\}$ is a nonincreasing sequence with nonnegative terms. As a result, there is a nonnegative constant $\rho$ such that $\lim _{n \rightarrow \infty} D\left(w_{n-1}, w_{n}\right)=\rho$. We shall show that $\rho>0$. Indeed, from (3.3), we derive that

$$
D\left(w_{n}, w_{n+1}\right) \leq \lambda D\left(w_{n-1}, w_{n}\right) \leq \lambda^{n} D\left(w_{0}, w_{1}\right) .
$$

The rest of the proof follows along the same lines as the proof of Theorem 2.3. 
Theorem 3.3 Consider an $\mathcal{F}$-complete $\mathcal{F}$-metric space $(A, D)$ and let $T$ be a symmetric fractional $\alpha-\eta$-contraction of type II satisfying the following conditions:

(i) $T$ is an $\alpha$-admissible mapping with respect to $\eta$;

(ii) there exists an $w_{0} \in A$ such that $\alpha\left(w_{0}, T w_{0}\right) \geq \eta\left(w_{0}, T w_{0}\right)$;

(iii) there is a sequence $\left\{w_{n}\right\}$ in $A$ such that $\alpha\left(w_{n}, w_{n+1}\right) \geq \eta\left(w_{n}, w_{n+1}\right), w_{n} \rightarrow w^{*}$ as $n \rightarrow \infty$, and $\alpha\left(w_{n}, w^{*}\right) \geq \eta\left(w_{n}, w^{*}\right)$ holds for each $n \in \mathbb{N}$.

Then, $T$ possesses a fixed point in $A$.

Proof We follow the lines of the proof of Theorem 2.4. Since, by (iii), $\alpha\left(w_{n}, w^{*}\right) \geq \eta\left(w_{n}, w^{*}\right)$ holds for every $n \in \mathbb{N}$, using $\left(D_{3}\right)$, we get

$$
f\left(D\left(T w^{*}, w^{*}\right)\right) \leq f\left(D\left(T w^{*}, T w_{n}\right)+D\left(w_{n}, w^{*}\right)\right)+\mu .
$$

From (3.1) and $\left(\mathcal{F}_{1}\right)$, we have

$$
\begin{aligned}
& f\left(D\left(T w^{*}, w^{*}\right)\right) \leq f\left(\left(D\left(T w^{*}, T w_{n}\right)\right)+D\left(T w_{n}, w^{*}\right)\right)+\mu \\
& \leq f\left(\lambda\left(\begin{array}{c}
D\left(w^{*}, w_{n}\right) \cdot D\left(T w^{*}, w^{*}\right)^{\frac{\beta}{(\beta-\hat{w})(\beta-\gamma)}} \cdot D\left(w_{n}, T w_{n}\right)^{\frac{\beta}{(\beta-\hat{w})(\beta-\gamma)}} \\
\cdot\left[D\left(T w^{*}, w^{*}\right)+D\left(w_{n}, T w_{n}\right)\right]^{\frac{\hat{w}}{(\hat{w}-\beta)(\hat{w}-\gamma)}} \\
\cdot\left[D\left(T w_{n}, w^{*}\right)+D\left(w_{n}, T w^{*}\right)\right]^{\frac{\gamma}{(\gamma-\beta)(\gamma-\hat{w})}}+D\left(w_{n+1}, w^{*}\right)
\end{array}\right)\right) \\
&+\mu .
\end{aligned}
$$

Making use of (2.6) and that

$$
\lim _{n \rightarrow \infty} D\left(w_{n}, w^{*}\right)=0 \quad \text { together } \lim _{n \rightarrow \infty} D\left(w_{n+1}, w^{*}\right)=0,
$$

we obtain

$$
f\left(D\left(w^{*}, T w^{*}\right)\right) \leq f\left(D\left(w^{*}, T w^{*}\right)\right)+\mu .
$$

Using $\left(\mathcal{F}_{2}\right)$, we have

$$
\lim _{n \rightarrow \infty} f\left(D\left(w^{*}, T w^{*}\right)\right)+\mu=-\infty,
$$

which is a logical inconsistency. Hence $D\left(w^{*}, T w^{*}\right)=0$, that is, $w^{*}$ is a fixed point of $T$.

\section{Symmetric fractional $\alpha-\eta$-contraction of type III}

In this section, a symmetric fractional $\alpha-\eta$-contraction of type III is considered in the setting of an $\mathcal{F}$-complete $\mathcal{F}$-metric space. Before stating a fixed-point theorem for such maps, we define a symmetric fractional $\alpha-\eta$-contraction of type III as follows:

Definition 4.1 Let $(A, D)$ be an $\mathcal{F}$-metric space with a self-mapping $T: A \rightarrow A$ and two functions $\alpha, \eta: A \times A \rightarrow[0,+\infty)$. We say that $T$ is a symmetric fractional $\alpha-\eta$-contraction of type III if there are constants $\lambda \in[0,1)$ and $\beta, \hat{w}, \gamma \in(0,1)$ such that, whenever $\alpha(w, v) \geq$ $\eta(w, v)$, we have

$$
D(T w, T v) \leq \lambda\left(\check{S}_{3}(w, v)\right)
$$


where

$$
\check{S}_{3}(w, v)=\lambda \max \left\{\begin{array}{c}
D(w, v),[D(w, T w)]^{\frac{\beta^{2}}{(\beta-\hat{w})(\beta-\gamma)}} \cdot[D(v, T v)]^{\frac{\beta^{2}}{(\beta-\hat{w})(\beta-\gamma)}} \\
\cdot[D(w, T w)+D(v, T v)]^{\frac{\hat{w}^{2}}{(\hat{w}-\beta)(\hat{w}-\gamma)}} \\
\cdot[D(w, T v)+D(v, T w)]^{\frac{\gamma^{2}}{(\gamma-\beta)(\gamma-\hat{\psi})}}
\end{array}\right\},
$$

for all $w, v \in A \backslash \operatorname{Fix}(T)$.

Now we state and prove our next theorem.

Theorem 4.2 Let $(A, D)$ be an $\mathcal{F}$-complete $\mathcal{F}$-metric space and consider a symmetric fractional $\alpha-\eta$-contraction $T$ of type III that satisfies the following conditions:

(i) $T$ is a $\alpha$-admissible mapping with respect to $\eta$;

(ii) there exist a $w_{0} \in A$ such that $\alpha\left(w_{0}, T w_{0}\right) \geq \eta\left(w_{0}, T w_{0}\right)$;

(iii) $T$ is $\alpha-\eta$-continuous.

Then, $T$ possesses a fixed point in $A$.

Proof Let $w_{0}$ in $A$ be such that $\alpha\left(w_{0}, T w_{0}\right) \geq \eta\left(w_{0}, T w_{0}\right)$. Using this $w_{0} \in A$, we define $\left\{w_{n}\right\}_{n=1}^{\infty}$ in such a way that $w_{1}=T w_{0}, w_{2}=T w_{1}=T^{2} w_{0}$. Continuing this way, we get $w_{n+1}=T w_{n}=T^{n+1} w_{0}$, for every $n \in \mathbb{N}$. Since the mapping $T$ is $\alpha$-admissible with respect to $\eta, \alpha\left(w_{0}, w_{1}\right)=\alpha\left(w_{0}, T w_{0}\right) \geq \eta\left(w_{0}, T w_{0}\right)=\eta\left(w_{0}, w_{1}\right)$. Carrying on in this way, we find

$$
\alpha\left(w_{n-1}, w_{n}\right) \geq \eta\left(w_{n-1}, w_{n}\right)=\eta\left(w_{n-1}, T w_{n-1}\right), \quad \text { for all } n \in \mathbb{N} .
$$

If $w_{n+1}=w_{n}$ for some $n \in \mathbb{N}$ then $w_{n}=w^{*}$ is a fixed point of $T$. So, we assume that $w_{n} \neq w_{n+1}$ and that

$$
D\left(T w_{n-1}, T w_{n}\right)=D\left(w_{n}, T w_{n}\right)>0, \quad \text { for each } n \in \mathbb{N} .
$$

As $T$ is a symmetric fractional $\alpha-\eta$-contraction of type III, for any $n \in \mathbb{N}$, we get

$$
\begin{aligned}
D\left(w_{n}, w_{n+1}\right)= & D\left(T w_{n-1}, T w_{n}\right) \\
\leq & \lambda \max \left[D\left(w_{n-1}, w_{n}\right), D\left(w_{n-1}, T w_{n-1} \frac{\beta^{2}}{(\beta-\hat{w})(\beta-\gamma)}\right.\right. \\
& \cdot D\left(w_{n}, T w_{n}\right)^{\frac{\beta^{2}}{(\beta-\hat{w})(\beta-\gamma)}} \\
& \cdot\left[D\left(w_{n-1}, T w_{n-1}\right)+D\left(w_{n}, T w_{n}\right)\right]^{\frac{\hat{w}^{2}}{(\hat{w}-\beta)(\hat{w}-\gamma)}} \\
& \left.\cdot\left[D\left(w_{n-1}, T w_{n}\right)+D\left(w_{n}, T w_{n-1}\right)\right]^{\frac{\gamma^{2}}{(\gamma-\beta)(\gamma-\hat{w})}}\right] \\
= & \lambda \max \left[D\left(w_{n-1}, w_{n}\right), D\left(w_{n-1}, w_{n}\right)^{\frac{\beta^{2}}{(\beta-\hat{w})(\beta-\gamma)}} \cdot D\left(w_{n}, w_{n+1}\right)^{\frac{\beta^{2}}{(\beta-\hat{w})(\beta-\gamma)}}\right. \\
& \cdot\left[D\left(w_{n-1}, w_{n}\right)+D\left(w_{n}, w_{n+1}\right)\right]^{\frac{\hat{\hat{w}}^{2}}{(\hat{w}-\beta)(\hat{w}-\gamma)}} \\
& \left.\cdot\left[D\left(w_{n-1}, w_{n+1}\right)+D\left(w_{n}, w_{n}\right)\right]^{\frac{\gamma^{2}}{(\gamma-\beta)(\gamma-\hat{w})}}\right]
\end{aligned}
$$




$$
\begin{aligned}
\leq & \lambda \max \left[D\left(w_{n-1}, w_{n}\right), D\left(w_{n-1}, w_{n}\right)^{\frac{\beta^{2}}{(\beta-\hat{w})(\beta-\gamma)}} \cdot D\left(w_{n}, w_{n+1}\right)^{\frac{\beta^{2}}{(\beta-\hat{w})(\beta-\gamma)}}\right. \\
& \cdot\left[D\left(w_{n-1}, w_{n}\right)+D\left(w_{n}, w_{n+1}\right)\right]^{\frac{\hat{w}^{2}}{(\hat{w}-\beta)(\hat{w}-\gamma)}} \\
& \left.\cdot\left[D\left(w_{n-1}, w_{n}\right)+D\left(w_{n}, w_{n+1}\right)\right]^{\frac{\gamma^{2}}{(\gamma-\beta)(\gamma-\hat{w})}}\right] \\
= & \lambda \max \left[D\left(w_{n-1}, w_{n}\right), D\left(w_{n-1}, w_{n}\right)^{\frac{\beta^{2}}{(\beta-\hat{w})(\beta-\gamma)}} \cdot D\left(w_{n}, w_{n+1}\right)^{\frac{\beta^{2}}{(\beta-\hat{w})(\beta-\gamma)}}\right. \\
& \left.\cdot\left[D\left(w_{n-1}, w_{n}\right)+D\left(w_{n}, w_{n+1}\right)\right]^{\frac{\hat{w}^{2}}{(\hat{w}-\beta)(\hat{w}-\gamma)}+\frac{\gamma^{2}}{(\gamma-\beta)(\gamma-\hat{w})}}\right] \\
\leq & \lambda \max \left[D\left(w_{n-1}, w_{n}\right), D\left(w_{n-1}, w_{n}\right)^{\frac{\beta^{2}}{(\beta-\hat{w})(\beta-\gamma)}} \cdot D\left(w_{n}, w_{n+1}\right)^{\frac{\beta^{2}}{(\beta-\hat{w})(\beta-\gamma)}}\right. \\
& \left.\cdot\left[D\left(w_{n-1}, w_{n}\right) \cdot D\left(w_{n}, w_{n+1}\right)\right]^{\frac{\hat{w}^{2}}{(\hat{w}-\beta)(\hat{w}-\gamma)}+\frac{\gamma^{2}}{(\gamma-\beta)(\gamma-\hat{w})}}\right] \\
= & \lambda \max \left[D\left(w_{n-1}, w_{n}\right), D\left(w_{n-1}, w_{n}\right)^{\frac{\beta^{2}}{(\beta-\hat{w})(\beta-\gamma)}+\frac{\hat{w}^{2}}{(\hat{w}-\beta)(\hat{w}-\gamma)}+\frac{\gamma^{2}}{(\gamma-\beta)(\gamma-\hat{w})}}\right. \\
& \left.\cdot D\left(w_{n}, w_{n+1}\right)^{\frac{\beta^{2}}{(\beta-\hat{w})(\beta-\gamma)}+\frac{\hat{w}^{2}}{(\hat{w}-\beta)(\hat{w}-\gamma)}+\frac{\gamma^{2}}{(\gamma-\beta)(\gamma-\hat{w})}}\right] \\
= & \lambda \max \left\{D\left(w_{n-1}, w_{n}\right), D\left(w_{n}, w_{n+1}\right)\right\} .
\end{aligned}
$$

If $\max \left\{D\left(w_{n}, w_{n+1}\right), D\left(w_{n-1}, w_{n}\right)\right\}=D\left(w_{n}, w_{n+1}\right)$ then

$$
D\left(w_{n}, w_{n+1}\right) \leq \lambda D\left(w_{n}, w_{n+1}\right),
$$

which is a contradiction. Thus we deduce that

$$
D\left(w_{n}, w_{n+1}\right) \leq \lambda D\left(w_{n-1}, w_{n}\right),
$$

and conclude that $\left\{D\left(w_{n-1}, w_{n}\right)\right\}$ is a nonincreasing sequence with nonnegative terms. So there is a nonnegative constant $\rho$ such that $\lim _{n \rightarrow \infty} D\left(w_{n-1}, w_{n}\right)=\rho$. We shall establish that $\rho>0$. Indeed, from (4.3), we derive that

$$
D\left(w_{n}, w_{n+1}\right) \leq \lambda D\left(w_{n-1}, w_{n}\right) \leq \lambda^{n} D\left(w_{0}, w_{1}\right)
$$

The rest of the argument follows the lines of the proof of Theorem 2.3.

Theorem 4.3 Consider an $\mathcal{F}$-complete $\mathcal{F}$-metric space $(A, D)$ and let $T$ be a symmetric fractional $\alpha-\eta$-contraction of type III satisfying the following conditions:

(i) $T$ is an $\alpha$-admissible mapping with respect to $\eta$;

(ii) there exists an $w_{0} \in A$ such that $\alpha\left(w_{0}, T w_{0}\right) \geq \eta\left(w_{0}, T w_{0}\right)$;

(iii) there is a sequence $\left\{w_{n}\right\}$ in A such that $\alpha\left(w_{n}, w_{n+1}\right) \geq \eta\left(w_{n}, w_{n+1}\right), w_{n} \rightarrow w^{*}$ as $n \rightarrow \infty$, and that $\alpha\left(w_{n}, w^{*}\right) \geq \eta\left(w_{n}, w^{*}\right)$ holds for each $n \in \mathbb{N}$.

Then $T$ possesses a fixed point in $A$.

Proof Much as in the proof of Theorem 2.4, considering (iii), we have $\alpha\left(w_{n}, w^{*}\right) \geq$ $\eta\left(w_{n}, w^{*}\right)$ for all $n \in \mathbb{N}$. By $\left(D_{3}\right)$, we obtain

$$
f\left(D\left(T w^{*}, w^{*}\right)\right) \leq f\left(D\left(T w^{*}, T w_{n}\right)+D\left(w_{n}, w^{*}\right)\right)+\mu
$$


Using (3.1) along with $\left(\mathcal{F}_{1}\right)$, we have

$$
\begin{aligned}
& f\left(D\left(T w^{*}, w^{*}\right)\right) \leq f\left(\left(D\left(T w^{*}, T w_{n}\right)\right)+D\left(T w_{n}, w^{*}\right)\right)+\mu \\
& \leq f\left(\lambda\left(\begin{array}{c}
D\left(w^{*}, w_{n}\right), D\left(T w^{*}, w^{*}\right)^{\frac{\beta^{2}}{(\beta-\hat{w})(\beta-\gamma)}} \cdot D\left(w_{n}, T w_{n}\right)^{\frac{\beta^{2}}{(\beta-\hat{w})(\beta-\gamma)}} \\
\cdot\left[D\left(T w^{*}, w^{*}\right)+D\left(w_{n}, T w_{n}\right)\right]^{\frac{\hat{w}^{2}}{(\hat{w}-\hat{\beta})(\hat{w}-\gamma)}} \\
\cdot\left[D\left(T w_{n}, w^{*}\right)+D\left(w_{n}, T w^{*}\right)\right]^{\frac{\gamma^{2}}{(\gamma-\beta)(\gamma-\hat{w})}}+D\left(w_{n+1}, w^{*}\right)
\end{array}\right)\right) \\
&+\mu .
\end{aligned}
$$

Using (2.6) implies

$$
\lim _{n \rightarrow \infty} D\left(w_{n}, w^{*}\right)=0 \quad \text { as long as } \quad \lim _{n \rightarrow \infty} D\left(w_{n+1}, w^{*}\right)=0
$$

so we obtain

$$
f\left(D\left(w^{*}, T w^{*}\right)\right) \leq f\left(D\left(w^{*}, T w^{*}\right)\right)+\mu .
$$

Utilizing $\left(\mathcal{F}_{2}\right)$, we have

$$
\lim _{n \rightarrow \infty} f\left(D\left(w^{*}, T w^{*}\right)\right)+\mu=-\infty,
$$

which is a logical inconsistency. Hence $D\left(w^{*}, T w^{*}\right)=0$, that is, $w^{*}$ a fixed point of $T$.

\section{Symmetric fractional $\alpha-\eta$-contraction of type IV}

In this part, we propose a new notion of symmetric fractional $\alpha-\eta$-contraction of type IV in the framework of an $\mathcal{F}$-complete $\mathcal{F}$-metric space.

Definition 5.1 Consider an $\mathcal{F}$-metric space $(A, D)$ with a self-mapping $T: A \rightarrow A$ and two functions $\alpha, \eta: A \times A \rightarrow[0,+\infty)$. We name $T$ a symmetric fractional $\alpha-\eta$-contraction of type IV if there are constants $\lambda \in[0,1)$ and $\beta, \hat{w}, \gamma \in(0,1)$ with $\beta+\hat{w}+\gamma<1$ such that, whenever $\alpha(w, v) \geq \eta(w, v)$, we have

$$
D(T w, T v) \leq \lambda\left(\check{S}_{4}(w, v)\right)
$$

where

$$
\check{S}_{4}(w, v)=\lambda\left\{\begin{array}{c}
D(w, v)^{\frac{\beta^{3}}{(\beta-\hat{w})(\beta-\gamma)}} \cdot D(w, T w)^{\frac{\beta^{3}}{(\beta-\hat{w})(\beta-\gamma)}} \cdot[D(w, T w)+D(v, T v)]^{\frac{\left.\hat{\hat{w}}^{3}-\beta\right)}{(\hat{w}-\gamma)}} \\
\cdot[D(w, T v)+D(v, T w)]^{\frac{\gamma^{3}}{(\gamma-\beta)(\gamma-\hat{w})}}
\end{array}\right\},
$$

for all $w, v \in A \backslash \operatorname{Fix}(T)$.

Now we state and prove our next theorem.

Theorem 5.2 Consider an $\mathcal{F}$-complete $\mathcal{F}$-metric space $(A, D)$ along with a symmetric fractional $\alpha-\eta$-contraction $T$ of type IV that satisfies the following conditions: 
(i) $T$ is a $\alpha$-admissible mapping with respect to $\eta$;

(ii) there exist a $w_{0} \in A$ such that $\alpha\left(w_{0}, T w_{0}\right) \geq \eta\left(w_{0}, T w_{0}\right)$;

(iii) $T$ is $\alpha-\eta$-continuous.

Then $T$ possesses a fixed point in $A$.

Proof Let $w_{0}$ in $A$ be such that $\alpha\left(w_{0}, T w_{0}\right) \geq \eta\left(w_{0}, T w_{0}\right)$. For this $w_{0} \in A$, we build a sequence $\left\{w_{n}\right\}_{n=1}^{\infty}$ in such a way that $w_{1}=T w_{0}, w_{2}=T w_{1}=T^{2} w_{0}$. Proceeding this way, $w_{n+1}=T w_{n}=T^{n+1} w_{0}$, for every $n \in \mathbb{N}$. Since the mapping $T$ is $\alpha$-admissible with respect to $\eta, \alpha\left(w_{0}, w_{1}\right)=\alpha\left(w_{0}, T w_{0}\right) \geq \eta\left(w_{0}, T w_{0}\right)=\eta\left(w_{0}, w_{1}\right)$. Carrying on in this way, we get

$$
\alpha\left(w_{n-1}, w_{n}\right) \geq \eta\left(w_{n-1}, w_{n}\right)=\eta\left(w_{n-1}, T w_{n-1}\right), \quad \text { for each } n \in \mathbb{N} .
$$

If $w_{n+1}=w_{n}$ for some $n \in \mathbb{N}$ then $w_{n}=w^{*}$ is a fixed point of $T$. So, we assume that $w_{n} \neq w_{n+1}$ and

$$
D\left(T w_{n-1}, T w_{n}\right)=D\left(w_{n}, T w_{n}\right)>0, \quad \text { for all } n \in \mathbb{N} .
$$

As $T$ is a symmetric fractional $\alpha-\eta$-contraction of type IV, for each $n \in \mathbb{N}$, we have

$$
\begin{aligned}
& D\left(w_{n}, w_{n+1}\right)=D\left(T w_{n-1}, T w_{n}\right) \\
& \leq \lambda\left[D\left(w_{n-1}, w_{n}\right)^{\frac{\beta^{3}}{(\beta-\hat{)}(\beta-\gamma)}} \cdot D\left(w_{n-1}, T w_{n-1}\right)^{\frac{\beta^{3}}{(\beta-\hat{)}(\beta-\gamma)}}\right. \\
& \cdot\left[D\left(w_{n-1}, T w_{n-1}\right)+D\left(w_{n}, T w_{n}\right)\right]^{\frac{\hat{w}^{3}}{(\hat{w}-\beta)(\hat{w}-\gamma)}} \\
& \left.\cdot\left[D\left(w_{n-1}, T w_{n}\right)+D\left(w_{n}, T w_{n-1}\right)\right]^{\frac{\gamma^{3}}{(\gamma-\beta)(\gamma-\hat{w})}}\right] \\
& =\lambda\left[D\left(w_{n-1}, w_{n}\right)^{\frac{\beta^{3}}{(\beta-\hat{w})(\beta-\gamma)}} \cdot D\left(w_{n}, w_{n+1}\right)^{\frac{\beta^{3}}{(\beta-\hat{w})(\beta-\gamma)}}\right. \\
& \cdot\left[D\left(w_{n-1}, w_{n}\right)+D\left(w_{n}, w_{n+1}\right)\right]^{\frac{\hat{\hat{w}}^{3}}{(\hat{w}-\beta)(\hat{w}-\gamma)}} \\
& \left.\cdot\left[D\left(w_{n-1}, w_{n+1}\right)+D\left(w_{n}, w_{n}\right)\right]^{\frac{\gamma^{3}}{(\gamma-\beta)(\gamma-\hat{w})}}\right] \\
& \leq \lambda\left[D\left(w_{n-1}, w_{n}\right)^{\frac{\beta^{3}}{(\beta-\hat{w})(\beta-\gamma)}} \cdot D\left(w_{n}, w_{n+1}\right)^{\frac{\beta^{3}}{(\beta-\hat{w})(\beta-\gamma)}}\right. \\
& \cdot\left[D\left(w_{n-1}, w_{n}\right)+D\left(w_{n}, w_{n+1}\right)\right]^{\frac{\hat{\hat{w}}^{3}}{(\hat{w}-\beta)(\hat{w}-\gamma)}} \\
& \left.\cdot\left[D\left(w_{n-1}, w_{n}\right)+D\left(w_{n}, w_{n+1}\right)\right]^{\frac{\gamma^{3}}{\gamma-\beta)(\gamma-\hat{w})}}\right] \\
& =\lambda\left[D\left(w_{n-1}, w_{n}\right)^{\frac{\beta^{3}}{(\beta-\hat{w})(\beta-\gamma)}} \cdot D\left(w_{n}, w_{n+1}\right)^{\frac{\beta^{3}}{(\beta-\hat{w})(\beta-\gamma)}}\right. \\
& \left.\cdot\left[D\left(w_{n-1}, w_{n}\right)+D\left(w_{n}, w_{n+1}\right)\right]^{\frac{\hat{\hat{w}}^{3}}{(\hat{w}-\beta)(\hat{w}-\gamma)}+\frac{\gamma^{3}}{(\gamma-\beta)(\gamma-\hat{w})}}\right] \\
& \leq \lambda\left[\begin{array}{c}
D\left(w_{n-1}, w_{n}\right)^{\frac{\beta^{3}}{(\beta-\hat{w})(\beta-\gamma)}} \cdot D\left(w_{n}, w_{n+1}\right)^{\frac{\beta^{3}}{(\beta-\hat{w})(\beta-\gamma)}} \\
\cdot\left[D\left(w_{n-1}, w_{n}\right) \cdot D\left(w_{n}, w_{n+1}\right)\right]^{\frac{\hat{\hat{w}}^{3}}{(\hat{w}-\beta)(\hat{w}-\gamma)}+\frac{\gamma^{3}}{(\gamma-\beta)(\gamma-\hat{w})}}
\end{array}\right] \\
& =\lambda\left[D\left(w_{n-1}, w_{n}\right)^{\frac{\beta^{3}}{(\beta-\hat{w})(\beta-\gamma)}+\frac{\hat{\hat{w}}^{3}}{(\hat{w}-\beta)(\hat{w}-\gamma)}+\frac{\gamma^{3}}{(\gamma-\beta)(\gamma-\hat{w})}}\right.
\end{aligned}
$$




$$
\begin{aligned}
& \left.\cdot D\left(w_{n}, w_{n+1}\right) \frac{\beta^{3}}{(\beta-\hat{w})(\beta-\gamma)}+\frac{\hat{w}^{3}}{(\hat{w}-\beta)(\hat{w}-\gamma)}+\frac{\gamma^{3}}{(\gamma-\beta)(\gamma-\hat{w})}\right] \\
= & \lambda\left\{D\left(w_{n-1}, w_{n}\right) \cdot D\left(w_{n}, w_{n+1}\right)\right\}^{\beta+\hat{w}+\gamma} \\
\leq & \lambda \max \left\{D\left(w_{n-1}, w_{n}\right), D\left(w_{n}, w_{n+1}\right)\right\} .
\end{aligned}
$$

If $\max \left\{D\left(w_{n}, w_{n+1}\right), D\left(w_{n-1}, w_{n}\right)\right\}=D\left(w_{n}, w_{n+1}\right)$ then

$$
D\left(w_{n}, w_{n+1}\right) \leq \lambda D\left(w_{n}, w_{n+1}\right),
$$

which is a contradiction. So we deduce that

$$
D\left(w_{n}, w_{n+1}\right) \leq \lambda D\left(w_{n-1}, w_{n}\right) .
$$

The rest of the argument follows the proof of Theorem 2.3.

Theorem 5.3 Consider an $\mathcal{F}$-complete $\mathcal{F}$-metric space $(A, D)$ and let $T$ be a symmetric fractional $\alpha-\eta$-contraction of type IV fulfilling the following conditions:

(i) $T$ is a $\alpha$-admissible mapping with respect to $\eta$;

(ii) there exists a $w_{0} \in A$ such that $\alpha\left(w_{0}, T w_{0}\right) \geq \eta\left(w_{0}, T w_{0}\right)$;

(iii) there is a sequence $\left\{w_{n}\right\}$ in A satisfying $\alpha\left(w_{n}, w_{n+1}\right) \geq \eta\left(w_{n}, w_{n+1}\right)$, such that $w_{n} \rightarrow w^{*}$ as $n \rightarrow \infty$, and $\alpha\left(w_{n}, w^{*}\right) \geq \eta\left(w_{n}, w^{*}\right)$ holds for each $n \in \mathbb{N}$.

Then $T$ possesses a fixed point in $A$.

Taking $\eta(w, v)=1$ in Theorems 2.3, 2.4, 3.2, and 3.3, we obtain the following corollaries.

Corollary 5.4 Consider an $\mathcal{F}$-complete $\mathcal{F}$-metric space $(A, D)$ and let $T$ be a symmetric fractional $\alpha-\eta$-contraction of type I fulfilling the following conditions:

(i) $T$ is a $\alpha$-admissible mapping;

(ii) there exists a $w_{0} \in A$ such that $\alpha\left(w_{0}, T w_{0}\right) \geq 1$;

(iii) $T$ is $\alpha-\eta$-continuous.

Then $T$ has a fixed point in $A$.

Corollary 5.5 Consider an $\mathcal{F}$-complete $\mathcal{F}$-metric space $(A, D)$ and let $T$ be a symmetric fractional $\alpha-\eta$-contraction of type I fulfilling the following conditions:

(i) $T$ is a $\alpha$-admissible mapping;

(ii) there exists a $w_{0} \in A$ such that $\alpha\left(w_{0}, T w_{0}\right) \geq 1$;

(iii) there is a sequence $\left\{w_{n}\right\}$ in A satisfying $\alpha\left(w_{n}, w_{n+1}\right) \geq 1$, such that $w_{n} \rightarrow w^{*}$ as $n \rightarrow \infty$, and $\alpha\left(w_{n}, w^{*}\right) \geq 1$ holds for each $n \in \mathbb{N}$.

Then $T$ possesses a fixed point in $A$.

Corollary 5.6 Consider an $\mathcal{F}$-complete $\mathcal{F}$-metric space $(A, D)$ and let $T$ be a symmetric fractional $\alpha-\eta$-contraction of type II satisfying the following conditions:

(i) $T$ is a $\alpha$-admissible mapping;

(ii) there exists a $w_{0} \in A$ such that $\alpha\left(w_{0}, T w_{0}\right) \geq 1$;

(iii) $T$ is $\alpha-\eta$-continuous.

Then $T$ has a fixed point in $A$. 
Corollary 5.7 Consider an $\mathcal{F}$-complete $\mathcal{F}$-metric space $(A, D)$ and let $T$ be a symmetric fractional $\alpha-\eta$-contraction of type II satisfying the following conditions:

(i) $T$ is a $\alpha$-admissible mapping;

(ii) there exists a $w_{0} \in A$ such that $\alpha\left(w_{0}, T w_{0}\right) \geq 1$;

(iii) there is a sequence $\left\{w_{n}\right\}$ in A satisfying $\alpha\left(w_{n}, w_{n+1}\right) \geq 1$ such that $w_{n} \rightarrow w^{*}$ as $n \rightarrow \infty$, and $\alpha\left(w_{n}, w^{*}\right) \geq 1$ holds for each $n \in \mathbb{N}$.

Then $T$ possesses a fixed point in $A$.

In a similar fashion, we can deduce analogues of Corollaries 5.4, 5.5, 5.6, and 5.7 for a symmetric fractional $\alpha-\eta$-contraction of type III and IV, respectively.

\section{Consequences}

As a consequence of our results, we derive some results for Suzuki-type contractions, orbitally $T$-complete and orbitally continuous mappings in $\mathcal{F}$-metric spaces.

Theorem 6.1 Consider an $\mathcal{F}$-metric space $(A, D)$ and let $T$ be a continuous self-mapping on $A$. Assume that there are $r \in[0,1)$ and $\beta, \hat{w}, \gamma \in(0,1)$ such that

$$
D(w, T w) \leq D(w, v)) \text { implies } D(T w, T v) \leq r\left(\check{S}_{1}(w, v)\right)
$$

where

$$
\begin{aligned}
\check{S}_{1}(w, v)= & D(w, v) \cdot D(w, T w)^{\frac{1}{(\beta-\hat{w})(\beta-\gamma)}} \cdot D(v, T v)^{\frac{1}{(\beta-\hat{w})(\beta-\gamma)}} \\
& \cdot[D(w, T w)+D(v, T v)]^{\frac{1}{(\hat{w}-\beta)(\hat{w}-\gamma)}} \cdot[D(w, T v)+D(v, T w)]^{\frac{1}{(\gamma-\beta)(\gamma-\hat{w})}}
\end{aligned}
$$

for all $w, v \in A \backslash \operatorname{Fix}(T)$.

Then $T$ possesses a fixed point in $A$.

Proof Describe $\alpha, \eta: A \times A \rightarrow[0,+\infty)$ by

$$
\alpha(w, v)=D(w, v) \quad \text { and } \quad \eta(w, v)=D(w, T w), \quad \text { for all } w, v \in A,
$$

and $\beta, \hat{w}, \gamma \in(0,1)$, as well as $r \in[0,1)$. It is clear that

$$
\eta(w, v) \leq \alpha(w, v), \quad \text { for all } w, v \in A,
$$

that is, conditions (i)-(iii) of our Theorem 2.3 hold true. If

$$
\eta(w, T w) \leq \alpha(w, v) \quad \text { then } D(w, T w) \leq D(w, v),
$$

which implies the contractive condition

$$
D(T w, T v) \leq r\left(\check{S}_{1}(w, v)\right) .
$$

Finally, every assumption of Theorem 2.3 holds true. Hence $T$ possesses a fixed point in $A$. 
Theorem 6.2 Consider an $\mathcal{F}$-metric space $(A, D)$ and let $T$ be a self-mapping of $A$. Suppose the following assumptions hold:

(i) $(A, D)$ is an orbitally $T$-complete $\mathcal{F}$-metric space;

(ii) there exist $r \in[0,1)$ and $\beta, \hat{w}, \gamma \in(0,1)$ such that

$$
D(T w, T v) \leq r\left(\check{S}_{1}(w, v)\right)
$$

where

$$
\begin{aligned}
\check{S}_{1}(w, v)= & D(w, v) \cdot D(w, T w)^{\frac{1}{(\beta-\hat{w}(\beta-\gamma)}} \cdot D(v, T v)^{\frac{1}{(\beta-\hat{w})(\beta-\gamma)}} \\
& \cdot[D(w, T w)+D(v, T v)]^{\frac{1}{(\hat{w}-\beta)(\hat{w}-\gamma)}} \cdot[D(w, T v)+D(v, T w)]^{\frac{1}{(\gamma-\beta)(\gamma-\hat{w})}}
\end{aligned}
$$

for all $w, v \in O(\omega)$ for some $\omega \in A$, where $O(\omega)$ is an orbit of $\omega$;

(iii) if $\left\{v_{n}\right\}$ is a sequence such that $\left\{v_{n}\right\} \subseteq O(\omega)$ with $v_{n} \rightarrow v^{*}$ as $n \rightarrow \infty$ then $v^{*} \in O(\omega)$. Then $T$ possesses a fixed point.

Proof Describe $\alpha, \eta: A \times A \rightarrow[0,+\infty)$ by setting $\alpha(w, v)=3$ on $O(\omega) \times O(\omega)$ and $\alpha(w, v)=$ 0 otherwise, and $\eta(w, v)=1$ for all $w, v \in A$ (see [7, Remark 6]). Then $(A, D)$ is an $\alpha-\eta$ complete $\mathcal{F}$-metric space and $T$ is an $\alpha$-admissible mapping with respect to $\eta$. If $\alpha(w, v) \geq$ $\eta(w, v)$ then $w, v \in O(\omega)$, and so from (ii) we have

$$
D(T w, T v) \leq r\left(\check{S}_{1}(w, v)\right)
$$

where

$$
\begin{aligned}
\check{S}_{1}(w, v)= & D(w, v) \cdot D(w, T w)^{\frac{1}{(\beta-\hat{w})(\beta-\gamma)}} \cdot D(v, T v)^{\frac{1}{(\beta-\hat{w})(\beta-\gamma)}} \\
& \cdot[D(w, T w)+D(v, T v)]^{\frac{1}{(\hat{w}-\beta)(\hat{w}-\gamma)}} \cdot[D(w, T v)+D(v, T w)]^{\frac{1}{(\gamma-\beta)(\gamma-\hat{w})}},
\end{aligned}
$$

that is, $T$ is a symmetric fractional $\alpha-\eta$-contraction of type I. Let $\left\{v_{n}\right\}$ be a sequence such that $\alpha\left(v_{n}, v_{n+1}\right) \geq \eta\left(v_{n}, v_{n+1}\right)$ and $v_{n} \rightarrow v^{*}$ for $n \rightarrow \infty$. So, $\left\{v_{n}\right\} \subseteq O(\omega)$. From (iii), $v^{*} \in$ $O(\omega)$, that is, $\alpha\left(v_{n}, v^{*}\right) \geq \eta\left(v_{n}, v^{*}\right)$. Hence every assumption of Theorem 2.4 holds true. Thus $T$ possesses a fixed point.

Theorem 6.3 Consider an $\mathcal{F}$-metric space $(A, D)$ and let $T$ be a self-mapping of $A$. Suppose the following conditions hold:

(i) for all $w, v \in O(\omega)$, there are $r \in[0,1)$ and $\beta, \hat{w}, \gamma \in(0,1)$ such that

$$
D(T w, T v) \leq r\left(\check{S}_{1}(w, v)\right)
$$

where

$$
\begin{aligned}
\check{S}_{1}(w, v)= & D(w, v) \cdot[D(w, T w)]^{\frac{1}{(\hat{\beta-\hat{w}})(\beta-\gamma)}} \cdot[D(v, T v)]^{\frac{1}{(\beta-\hat{w})(\beta-\gamma)}} \\
& \cdot[D(w, T w)+D(v, T v)]^{\frac{1}{(\hat{w}-\beta)(\hat{w}-\gamma)}} \cdot[D(w, T v)+D(v, T w)]^{\frac{1}{(\gamma-\beta)(\gamma-\hat{w})}}
\end{aligned}
$$

for some $\omega \in A$; 
(ii) the operator $T$ is orbitally continuous.

Then $T$ possesses a fixed point.

Proof Describe $\alpha, \eta: A \times A \rightarrow[0,+\infty)$ by setting $\alpha(w, v)=3$ on $O(\omega) \times O(\omega)$ and $\alpha(w, v)=$ 0 otherwise, with $\eta(w, v)=1$ (see [38, Remark 1.1]). We know that $T$ is an $\alpha-\eta$-continuous mapping. If $\alpha(w, v) \geq \eta(w, v)$ then $w, v \in O(\omega)$. So $T w, T v \in O(\omega)$, that is, $\alpha(T w, T v) \geq$ $\eta(T w, T v)$. Therefore $T$ is an $\alpha$-admissible mapping with respect to $\eta$. From (i) we have

$$
D(T w, T v) \leq r\left(\check{S}_{1}(w, v)\right)
$$

where

$$
\begin{aligned}
\check{S}_{1}(w, v)= & D(w, v) \cdot D(w, T w)^{\frac{1}{(\beta-\hat{w})(\beta-\gamma)}} \cdot D(v, T v)^{\frac{1}{(\beta-\hat{w})(\beta-\gamma)}} \\
& \cdot[D(w, T w)+D(v, T v)]^{\frac{1}{(\hat{w}-\beta)(\hat{w}-\gamma)}} \cdot[D(w, T v)+D(v, T w)]^{\frac{1}{(\gamma-\beta)(\gamma-\hat{w})}}
\end{aligned}
$$

implying that $T$ is a symmetric fractional $\alpha-\eta$-contraction of type I. Hence each assumption of Theorem 2.3 holds true. Thus $T$ has a fixed point.

Theorem 6.4 Consider an $\mathcal{F}$-metric space $(A, D)$ and let $T$ be a self-mapping of $A$. Suppose the following conditions hold:

(i) $(A, D)$ is an orbitally $T$-complete $\mathcal{F}$-metric space;

(ii) there exist $r \in[0,1)$ and $\beta, \hat{w}, \gamma \in(0,1)$ such that

$$
D(T w, T v) \leq r\left(\check{S}_{2}(w, v)\right)
$$

where

$$
\begin{aligned}
\check{S}_{2}(w, v)= & D(w, v) \cdot[D(w, T w)]^{\frac{\beta}{(\beta-\hat{w})(\beta-\gamma)}} \cdot[D(v, T v)]^{\frac{\beta}{(\beta-\hat{w})(\beta-\gamma)}} \\
& \cdot[D(w, T w)+D(v, T v)]^{\frac{\hat{w}}{(\hat{w}-\beta)(\hat{w}-\gamma)}} \cdot[D(w, T v)+D(v, T w)]^{\frac{\gamma}{(\gamma-\beta)(\gamma-\hat{w})}}
\end{aligned}
$$

for all $w, v \in O(\omega)$, for some $\omega \in A$, where $O(\omega)$ is an orbit of $\omega$;

(iii) if $\left\{v_{n}\right\}$ is a sequence such that $\left\{v_{n}\right\} \subseteq O(\omega)$ with $v_{n} \rightarrow v^{*}$ as $n \rightarrow \infty$ then $v^{*} \in O(\omega)$.

Then, $T$ possesses a fixed point.

Theorem 6.5 Consider an $\mathcal{F}$-metric space $(A, D)$ and let $T$ be a self-mapping of $A$. Suppose the following conditions hold:

(i) for all $w, v \in O(\omega)$, there exist $r \in[0,1)$ and $\beta, \hat{w}, \gamma \in(0,1)$ such that

$$
D(T w, T v) \leq r\left(\check{S}_{2}(w, v)\right)
$$

where

$$
\begin{aligned}
\check{S}_{2}(w, v)= & D(w, v) \cdot[D(w, T w)]^{\frac{\beta}{(\beta-\hat{w})(\beta-\gamma)}} \cdot[D(v, T v)]^{\frac{\beta}{(\beta-\hat{w})(\beta-\gamma)}} \\
& \cdot[D(w, T w)+D(v, T v)]^{\frac{\hat{w}}{(\hat{w}-\beta)(\hat{w}-\gamma)}} \cdot[D(w, T v)+D(v, T w)]^{\frac{\gamma}{(\gamma-\beta)(\gamma-\hat{w})}}
\end{aligned}
$$

for some $\omega \in A$; 
(ii) the operator $T$ is an orbitally continuous.

Then $T$ possesses a fixed point.

Theorems 6.1-6.3 can be derived easily for symmetric fractional contractions of type III and IV, respectively.

\section{Application to fractional differential equations}

The local and nonlocal fractional differential equations have recently proved to be significant tools in the modeling of many phenomena in numerous fields of science and engineering. The fractional-order differential equations have numerous applications in viscoelasticity, electrochemistry, control, porous media, electromagnetics, etc. For more details, see [9-25]. Our aim is to show the existence and uniqueness of a bounded solution to a boundary value problem involving a generalized fractional derivative in the RiemannLiouville sense.

Actually, the left Riemann-Liouville fractional integral of a Lebesgue-integrable function $f$ with respect to an increasing function $g$ is given as follows [10]:

$$
{ }_{a} I_{g}^{\alpha} f(t)=\frac{1}{\Gamma(\alpha)} \int_{a}^{t}(g(t)-g(s))^{\alpha-1} f(s) g^{\prime}(s) d s, \quad \text { where } \alpha>0 \text {. }
$$

The associated left Riemann-Liouville fractional derivative of $f$ with respect to the same increasing function $g$ is given by [10]

$$
\begin{aligned}
{ }_{a} D_{g}^{\alpha} f(t) & =\left(\frac{1}{g^{\prime}(t)} \frac{d}{d t}\right)^{n} I^{(n-\alpha)} f(t) \\
& =\left(\frac{1}{g^{\prime}(t)} \frac{d}{d t}\right)^{n} \frac{1}{\Gamma(\alpha)} \int_{a}^{t}(g(t)-g(s))^{n-\alpha-1} f(s) g^{\prime}(s) d s,
\end{aligned}
$$

where $\alpha \geq 0, n=[\alpha]+1$, and $[\alpha]$ is the integer part of $\alpha$. The following theorem combines the fractional integral and derivative.

Theorem 7.1 ([11]) Let $\alpha>0, n=-[-\alpha], f \in L[a, b]$, and ${ }_{a} I_{g}^{\alpha} f \in A C_{g}^{n}[a, b]$. Then

$$
{ }_{a} I_{g a}^{\alpha} D_{g}^{\alpha} f(t)=f(t)-\sum_{k=1}^{n} c_{k}(g(t)-g(a))^{\alpha-k}
$$

We are considering the following boundary value problem:

$$
{ }_{a} D_{g}^{\alpha} y(t)+f(t, y(t))=0, \quad y(a)=y(b)=0, \quad 1<\alpha \leq 2 .
$$

Lemma 7.2 Let $\alpha>0, n=-[-\alpha], f \in L[a, b]$, and ${ }_{a} I_{g}^{\alpha} f \in A C_{g}^{n}[a, b]$. Then, $y$ is a solution of the boundary value problem (7.3) if and only if

$$
y(t)=\int_{a}^{b} G(s, t) f(s, y(s)) g^{\prime}(s) d s
$$


where the Green's function

$$
G(s, t)=\frac{1}{\Gamma(\alpha)} \begin{cases}\left(\frac{(g(b)-g(s))(g(t)-g(a))}{(g(b)-g(a))}\right)^{\alpha-1}-(g(t)-g(s))^{\alpha-1}, & a<s \leq t, \\ \left(\frac{(g(b)-g(s))(g(t)-g(a))}{(g(b)-g(a))}\right)^{\alpha-1}, & t \leq s<b,\end{cases}
$$

satisfies the following:

- $G(s, t) \geq 0$

- $\max _{a \leq s, t \leq b} G(s, t)=\frac{1}{\Gamma(\alpha)}\left(\frac{g(b)-g(a)}{4}\right)^{\alpha-1}$.

Proof Applying the integral in (7.1) to (7.3), we get

$$
{ }_{a} I_{g a}^{\alpha} D_{g}^{\alpha} y(t)=-{ }_{a} I_{g}^{\alpha} f(t, y(t))=-\frac{1}{\Gamma(\alpha)} \int_{a}^{t}(g(t)-g(s))^{\alpha-1} f(s) g^{\prime}(s) d s .
$$

Now using Theorem 7.1, we obtain

$$
y(t)=c_{1}(g(t)-g(a))^{\alpha-1}+c_{2}(g(t)-g(a))^{\alpha-2}-\frac{1}{\Gamma(\alpha)} \int_{a}^{t}(g(t)-g(s))^{\alpha-1} f(s) g^{\prime}(s) d s .
$$

Then $y(a)=0$ gives $c_{2}=0$, while $y(b)=0$ gives

$$
c_{1}=\frac{(g(b)-g(a))^{1-\alpha}}{\Gamma(\alpha)} \int_{a}^{b}(g(b)-g(s))^{\alpha-1} f(s, y(s)) g^{\prime}(s) d s .
$$

Therefore

$$
\begin{aligned}
y(t)= & \frac{1}{\Gamma(\alpha)} \int_{a}^{b} \frac{(g(b)-g(s))(g(t)-g(a))^{\alpha-1}}{(g(b)-g(a))} f(s, y(s)) g^{\prime}(s) d s \\
& -\frac{1}{\Gamma(\alpha)} \int_{a}^{t}(g(t)-g(s))^{\alpha-1} f(s, y(s)) g^{\prime}(s) d s .
\end{aligned}
$$

Hence

$$
\begin{aligned}
& y(t)=\int_{a}^{b} G(s, t) f(s, y(s)) g^{\prime}(s) d s, \\
& \text { where } G(s, t)=\frac{1}{\Gamma(\alpha)} \begin{cases}\left(\frac{(g(b)-g(s))(g(t)-g(a))}{(g(b)-g(a))}\right)^{\alpha-1}-(g(t)-g(s))^{\alpha-1}, & a<s \leq t, \\
\left(\frac{(g(b)-g(s))(g(t)-g(a))}{(g(b)-g(a))}\right)^{\alpha-1}, & t \leq s<b .\end{cases}
\end{aligned}
$$

It is clear that $G(s, t) \geq 0$, when $s \geq t$.

For $a \leq s<t$, one can prove that

$$
\begin{aligned}
G(s, t)= & \left(\frac{(g(t)-g(a))}{(g(b)-g(a))}\right)^{\alpha-1} \\
& \cdot\left[(g(b)-g(a))^{\alpha-1}-\left(g(b)-\left(g(a)+\frac{(g(s)-g(a))(g(b)-g(a))}{(g(t)-g(a))}\right)^{\alpha-1}\right)\right] .
\end{aligned}
$$

Since $g(a)+\frac{(g(s)-g(a))(g(b)-g(a))}{(g(t)-g(a))} \geq g(s)$, one can deduce that $G(s, t) \geq 0$, for $s \leq t$. 
For $t \leq s$, we have

$$
\frac{\partial G}{\partial t}=\frac{1}{\Gamma(\alpha)}\left(\frac{g(b)-g(s)}{(g(b)-g(a))}\right)^{\alpha-1} \cdot(\alpha-1)(g(t)-g(a))^{\alpha-2} g^{\prime}(t) \geq 0,
$$

thus $G(s, t)$ is increasing as a function of $t$.

For $s \leq t$,

$$
\begin{aligned}
\frac{\partial G}{\partial t}= & \frac{g^{\prime}(t)(\alpha-1)}{\Gamma(\alpha)} \cdot\left[-(g(t)-g(s))^{\alpha-2}+\left(\frac{g(b)-g(s)}{g(b)-g(a)}\right)^{\alpha-1}(g(t)-g(a))^{\alpha-2}\right] \\
= & \frac{g^{\prime}(t)}{\Gamma(\alpha-1)} \cdot\left(\frac{g(t)-g(a)}{g(b)-g(a)}\right)^{\alpha-2} \\
& \cdot\left[\left(\frac{g(b)-g(s)}{g(b)-g(a)}\right)^{\alpha-1}-\left(\frac{(g(b)-g(a))(g(t)-g(s))}{g(b)-g(a)}\right)^{\alpha-2}\right] \\
\leq & \left.\frac{g^{\prime}(t)}{\Gamma(\alpha-1)} \cdot\left(\frac{g(t)-g(a)}{g(b)-g(a)}\right)^{\alpha-2}\right] \\
& \cdot\left[(g(b)-g(a))^{\alpha-2}-\left(g(b)-\left(g(a)+\frac{g(b)-g(a)}{g(t)-g(a)}(g(s)-g(a))\right)^{\alpha-2}\right)\right] \\
< & 0 .
\end{aligned}
$$

Thus $G(s, t)$ is decreasing when $s \leq t$. Hence $G(s, t)$ attains its maximum when $s=t$, and

$$
\begin{aligned}
G(s, s)= & \frac{1}{\Gamma(\alpha)} \frac{(g(b)-g(s))^{\alpha-1}(g(s)-g(a))^{\alpha-1}}{(g(b)-g(a))^{\alpha-1}}=\hat{G}(s) \\
\hat{G}^{\prime}(s)= & -\frac{1}{\Gamma(\alpha)}(\alpha-1) \frac{(g(b)-g(s))^{\alpha-2}}{(g(b)-g(a))^{\alpha-1}} g^{\prime}(s) \cdot(g(s)-g(a))^{\alpha-1} \\
& +\frac{1}{\Gamma(\alpha)} \frac{(g(b)-g(s))^{\alpha-1}(\alpha-1)(g(s)-g(a))^{\alpha-2} g^{\prime}(s)}{(g(b)-g(a))^{\alpha-1}} \\
= & 0
\end{aligned}
$$

yield $g(s)=\frac{g(a)+g(b)}{2}$, or that the critical point is

$$
s^{*}=g^{-1}\left(\frac{g(a)+g(b)}{2}\right) \text {. }
$$

Thus, the maximum of $G(s, t)$ is

$$
\begin{aligned}
& \hat{G}\left(s^{*}\right)=\frac{1}{\Gamma(\alpha)}\left(\frac{g(b)-g(a)}{4}\right)^{\alpha-1}, \\
& |G(s, t)| \leq \frac{1}{\Gamma(\alpha)}\left(\frac{g(b)-g(a)}{4}\right)^{\alpha-1} .
\end{aligned}
$$

Here we denote the Riemann-Stieltjes integrable function $w$ with respect to $s$ and $f$ : $[0,1] \times \mathbb{R} \rightarrow \mathbb{R}$ is a continuous function. Let $C(I)$ be the linear space of all continuous functions defined on $I=[0,1]$, and let $D(w, v)=\|w-v\|_{\infty}^{2}=\max _{t \in I}|w(t)-v(t)|^{2}$ for all $w, v \in C(I)$. Then $(C(I), D)$ is an $\mathcal{F}$-complete metric space. 
We consider the following conditions:

(a) there exist $r \in[0,1)$ and $\zeta: \mathbb{R}^{2} \rightarrow \mathbb{R}$, a function such that for each $a, b \in \mathbb{R}$ with $\zeta(a, b) \geq \xi(a, b)$ satisfies

$$
\begin{aligned}
& |f(s, w(s))-f(s, v(s))| \\
& \leq|w(s)-v(s)|^{2} \cdot|w(s)-T w(s)|^{\frac{2 \beta}{(\beta-\hat{w})(\beta-\gamma)}} \cdot|v(s)-T v(s)|^{\frac{2 \beta}{(\beta-\hat{w})(\beta-\gamma)}} \\
& \cdot[|w(s)-T w(s)|+|v(s)-T v(s)|]^{\frac{\hat{\hat{w}}}{(\hat{w}-\beta)(\hat{w}-\gamma)}} \\
& \cdot[|w(s)-T v(s)|+|v(s)-T w(s)|]^{\frac{\gamma}{(\gamma-\beta)(\gamma-\hat{w})},}
\end{aligned}
$$

where $\beta, \hat{w}, \gamma \in(0,1)$;

(b) there exists $w_{1} \in C(I)$ such that

$$
\zeta\left(w_{1}(t), \int_{a}^{b} G(t, s) f\left(s, w_{1}(s)\right) g^{\prime}(s) d s\right) \geq \xi\left(w_{1}(t), \int_{a}^{b} G(t, s) f\left(s, w_{1}(s)\right) g^{\prime}(s) d s\right)
$$

for all $t \in I$;

(c) for each $w, v \in C(I)$, there exist $w_{1}, v_{1} \in C(I)$ such that $\zeta(w(t), v(t)) \geq \xi(w(t), v(t))$ implies

$$
\begin{aligned}
& \zeta\left(\int_{a}^{b} G(t, s) f\left(s, w_{1}(s)\right) g^{\prime}(s) d s, \int_{a}^{b} G(t, s) f\left(s, v_{1}(s)\right) g^{\prime}(s) d s\right) \\
& \quad \geq \xi\left(\int_{a}^{b} G(t, s) f\left(s, w_{1}(s)\right) g^{\prime}(s) d s, \int_{a}^{b} G(t, s) f\left(s, v_{1}(s)\right) g^{\prime}(s) d s\right),
\end{aligned}
$$

for all $t \in I$;

(d) for any cluster point $w$ of a sequence $\left\{w_{n}\right\}$ of points in $C(I)$ with

$$
\zeta\left(w_{n}, w_{n+1}\right) \geq \xi\left(w_{n}, w_{n+1}\right), \quad \lim _{n \rightarrow \infty} \inf \zeta\left(w_{n}, w\right) \geq \lim _{n \rightarrow \infty} \inf \xi\left(w_{n}, w\right) .
$$

Theorem 7.3 Suppose that conditions (a)-(d) are satisfied. Then (7.3) has at least one solution $w^{*} \in C(I)$.

Proof We know that $w \in C(I)$ is a solution of (7.3) if and only if $w \in C(I)$ is a solution of the fractional-order integral equation

$$
w(t)=\lambda \int_{a}^{b} G(t, s) f(s, w(s)) g^{\prime}(s) d s \quad \text { for all } t \in I,
$$

where $\lambda, \in[0,1)$. We define a map $T: C(I) \rightarrow C(I)$ by

$$
T w(t)=\lambda \int_{a}^{b} G(t, s) f(s, w(s)) g^{\prime}(s) d s \quad \text { for all } t \in I
$$


Then problem (7.3) is equivalent to finding $w^{*} \in C(I)$ that is a fixed point of $T$. Let $w, v \in$ $C(I)$ be such that $\zeta(w(t), v(t)) \geq 0$ for all $t \in I$. For using condition (a), we get

$$
\begin{aligned}
& |T w(t)-T v(t)|=\left|\lambda \int_{a}^{b} G(t, s)[f(s, w(s))-f(s, v(s))] g^{\prime}(s) d s\right| \\
& \leq|\lambda| \int_{a}^{b}|G(t, s)|\left|f(s, w(s))-f(s, v(s)) g^{\prime}(s) d s\right| \\
& \leq|\lambda| \int_{a}^{b}|G(t, s)| g^{\prime}(s) r d s|w(s)-v(s)|^{2} \\
& \cdot|w(s)-T w(s)|^{\frac{2 \beta}{(\beta-\hat{w})(\beta-\gamma)}} \cdot|v(s)-T v(s)|^{\frac{2 \beta}{(\beta-\hat{w})(\beta-\gamma)}} \\
& \cdot[|w(s)-T w(s)|+|v(s)-T v(s)|]^{\frac{\hat{w}}{(\hat{w}-\beta)(\hat{w}-\gamma)}} \\
& \left.\cdot[|w(s)-T v(s)|+|v(s)-T w(s)|]^{\frac{\gamma}{(\gamma-\beta)(\gamma-\hat{w})}}\right] \\
& \leq \frac{1}{\Gamma(\alpha)}\left(\frac{g(b)-g(a)}{4}\right)^{\alpha-1}(g(b)-g(a))\|w(s)-v(s)\|_{\infty}^{2} \\
& \cdot\|w(s)-T w(s)\|_{\infty}^{\frac{2 \beta}{(\beta-\hat{w})(\beta-\gamma)}} \cdot\|v(s)-T v(s)\|_{\infty}^{\frac{2 \beta}{(\beta-\hat{w})(\beta-\gamma)}} \\
& \cdot\left[\|w(s)-T w(s)\|_{\infty}^{2}+\|v(s)-T v(s)\|_{\infty}^{2}\right]^{\frac{\hat{\hat{w}}-\beta)(\hat{w}-\gamma)}{\hat{w}}} \\
& \cdot\left[\|w(s)-T v(s)\|_{\infty}^{2}+\|v(s)-T w(s)\|_{\infty}^{2}\right]^{\frac{\gamma}{(\gamma-\beta)(\gamma-\hat{w})}} \\
& \leq r\|w(s)-v(s)\|_{\infty}^{2} \cdot\|w(s)-T w(s)\|_{\infty}^{\frac{2 \beta}{(\beta-\hat{)})(\beta-\gamma)}} \\
& \cdot\|v(s)-T v(s)\|_{\infty}^{\frac{2 \beta}{(\beta-\hat{)})(\beta-\gamma)}} \cdot\|w(s)-T v(s)\|_{\infty}^{2(p \gamma-q \gamma)} \\
& \cdot\left[\|w(s)-T w(s)\|_{\infty}^{2}+\|v(s)-T v(s)\|_{\infty}^{2}\right]^{\frac{\hat{\hat{w}}-\beta)(\hat{\mathcal{w}}-\gamma)}{\hat{w}}} \\
& \cdot\left[\|w(s)-T v(s)\|_{\infty}^{2}+\|v(s)-T w(s)\|_{\infty}^{2}\right]^{\frac{\gamma}{(\gamma-\beta)(\gamma-\hat{w})}} .
\end{aligned}
$$

Thus

$$
\begin{aligned}
D(T w, T v)< & |w(s)-v(s)|^{2} \cdot|w(s)-T w(s)|^{\frac{2 \beta}{(\beta-\hat{w})(\beta-\gamma)}} \\
& \cdot|v(s)-T v(s)|^{\frac{2 \beta}{(\beta-\hat{w})(\beta-\gamma)}} \cdot[|w(s)-T w(s)| \\
& +|v(s)-T v(s)|]^{\frac{\hat{w}}{(\hat{w}-\beta)(\hat{w}-\gamma)}} \cdot[|w(s)-T v(s)|+|v(s)-T w(s)|]^{\frac{\gamma}{(\gamma-\beta)(\gamma-\hat{w})}}
\end{aligned}
$$

for all $w, v \in C(I)$ such that $\zeta(w(t), v(t)) \geq \xi(w(t), v(t))$ for all $t \in I$. We define $\alpha: C(I) \times$ $C(I) \rightarrow[0, \infty)$ by

$$
\begin{aligned}
& \alpha(w, v)=\left\{\begin{array}{ll}
1 & \text { if } \zeta(w(t), v(t)) \geq 0, t \in I, \\
0 & \text { otherwise }
\end{array}\right. \text { and } \\
& \eta(w, v)= \begin{cases}\frac{1}{2} & \text { if } \xi(w(t), v(t)) \geq 0, t \in I, \\
0 & \text { otherwise. }\end{cases}
\end{aligned}
$$


Then, for all $w, v \in C(I), \alpha(w, v) \geq \eta(w, v)$, we have

$$
D(T w, T v) \leq r\left\{\begin{array}{c}
|w(s)-v(s)|^{2} \cdot|w(s)-T w(s)|^{\frac{2 \beta}{(\beta-\hat{w})(\beta-\gamma)}} \cdot|v(s)-T v(s)|^{\frac{2 \beta}{(\beta-\hat{w})(\beta-\gamma)}} \\
\cdot[|w(s)-T w(s)|+|v(s)-T v(s)|]^{\frac{\hat{w}}{(\hat{w}-\beta)(\hat{w}-\gamma)}} \\
\cdot[|w(s)-T v(s)|+|v(s)-T w(s)|]^{\frac{\gamma}{(\gamma-\beta)(\gamma-\hat{w})}} .
\end{array}\right.
$$

Obviously, $\alpha(w, v) \geq \eta(w, v)$ for all $w, v \in C(I)$. If $\alpha(w, v) \geq \eta(w, v)$ for each $w, v \in$ $C(I)$ then $\zeta(w(t), v(t)) \geq \xi(w(t), v(t))$. From condition (c), we have $\zeta(T w(t), T v(t)) \geq$ $\xi(T w(t), T v(t))$, and so $\alpha(T w, T v) \geq \eta(T w, T v)$. Thus, $T$ is an $\alpha$-admissible map with respect to $\eta$. From condition (b), there exists $w_{1} \in C(I)$ such that $\alpha\left(w_{1}, T w_{1}\right)=\eta\left(w_{1}, T w_{1}\right)$. By condition (d), we have that for any cluster point $w$ of a sequence $\left\{w_{n}\right\}$ of points in $C(I)$ with $\alpha\left(w_{n}, w_{n+1}\right)=\eta\left(w_{n}, w_{n+1}\right), \lim _{n \rightarrow \infty} \inf \alpha\left(w_{n}, w\right)=\lim _{n \rightarrow \infty} \inf \eta\left(w_{n}, w\right)$. By applying Theorem 2.3, $T$ has a fixed point in $C(I)$, i.e., there exists $w^{*} \in C(I)$ such that $T w^{*}=w^{*}$, and $w^{*}$ is a solution of (7.3).

Applications The fractional-order differential equations emerge in various areas of engineering and scientific disciplines as the mathematical modeling of systems and processes in the fields of physics, chemistry, control theory, biology, economics, blood flow phenomena, signal and image processing, biophysics, aerodynamics, fitting of experimental data.

\section{Conclusions}

The aim of this paper was to introduce four classes of symmetric fractional contraction. This research focuses on a new idea of symmetric fractional $\alpha-\eta$-contraction of type I, II, III, and IV in the setting of an $\mathcal{F}$-metric space, which is different and more general than an ordinary metric space. This paper will open a new domain of fixed-point theory. We develop here Suzuki-type fixed point results in orbitally complete $\mathcal{F}$-metric spaces. These new investigations and applications will enhance the impact of the new setup.

Acknowledgements

The authors thanks to their universities.

Funding

We declare that funding is not applicable for our paper.

Availability of data and materials

No data were used to support this study. It is not applicable for our paper.

Competing interests

The authors declare that they have no competing interests.

Authors' contributions

Writing, reviewing, and editing $\mathrm{AH}, \mathrm{FJ}$ and EK. All authors contributed equally and significantly in writing this article. All authors have read and agreed to the published version of the manuscript.

\section{Author details}

${ }^{1}$ Department of Mathematics, King Abdulaziz University, P.O. Box 80203, 21589 Jeddah, Saudi Arabia. ${ }^{2}$ Division of Applied Mathematics, Thu Dau Mot University, Binh Duong Province, Vietnam. ${ }^{3}$ Department of Mathematics, Cankaya University, Mimar Sinan Caddesi, 06790 Ankara, Turkey. ${ }^{4}$ Department of Medical Research, China Medical University Hospital, China Medical University, Hsueh-Shih Road, 40402 Taichung, Taiwan.

\section{Publisher's Note}

Springer Nature remains neutral with regard to jurisdictional claims in published maps and institutional affiliations. 


\section{References}

1. Reich, S.: Some problems and results in fixed point theory. Contemp. Math. 21, 179-185 (1983)

2. Kirk, W.A., Reich, S., Veeramani, P.: Proximinal retracts and best proximity pair theorems. Numer. Funct. Anal. Optim. 24(7), 851-862 (2003)

3. Jachymski, J.: The contraction principle for mappings on a metric space with a graph. Proc. Am. Math. Soc. 1(136), 1359-1373 (2008)

4. Bojor, F.: Fixed point theorems for Reich type contraction on metric spaces with a graph. Nonlinear Anal. 75, 3895-3901 (2012)

5. Abbas, M., Nazir, T.: Common fixed point of a power graphic contraction pair in partial metric spaces endowed with a graph. Fixed Point Theory Appl. 2013, 20 (2013)

6. Hussain, N., Al-Mezel, S., Salimi, P.: Fixed points for $\alpha-\psi$-graphic contractions with application to integral equations. Abstr. Appl. Anal. 2013, Article ID 575869 (2013)

7. Hussain, N., Kutbi, M.A., Salimi, P.: Fixed point theory in $\alpha$-complete metric spaces with applications. Abstr. Appl. Anal. 2014, Article ID 280817 (2014)

8. Jleli, M., Samet, B.: On a new generalization of metric spaces. J. Fixed Point Theory Appl. 20(3), 128 (2018)

9. Miller, K.S., Ross, B.: An Introduction to the Fractional Calculus and Fractional Differential Equations. Wiley, New York (1993)

10. Kilbas, A.A., Srivastava, H.M., Trujillo, J.J.: Theory and Applications of Fractional Differential Equations. North-Holland Mathematics Studies, vol. 204. Elsevier, Amsterdam (2006)

11. Jarad, F., Abdeljawad, T.: Generalized fractional derivatives and Laplace transform. Discrete Contin. Dyn. Syst., Ser. S 13(3), 709-722 (2020)

12. Baleanu, D., Agarwal, R.P., Mohammadi, H., Rezapour, S.: Some existence results for a nonlinear fractional differential equation on partially ordered Banach spaces. Bound. Value Probl. 2013, 112 (2013)

13. He, L., Dong, X., Bai, Z., Chen, B.: Solvability of some two-point fractional boundary value problems under barrier strip conditions. J. Funct. Spaces 2017, Article ID 1465623 (2017)

14. Hussain, A., Kanwal, T.: Existence and uniqueness for a neutral differential problem with unbounded delay via fixed point. Trans. A. Razmadze Math. Inst. 172(3), 480-490 (2018)

15. Jarad, F., Abdeljawad, T., Hammouch, Z.: On a class of ordinary differential equations in the frame of Atangana-Baleanu fractional derivative. Chaos Solitons Fractals 117, 16-20 (2018)

16. Abdeljawad, T., Agarwal, R.P., Karapinar, E., Kumari, P.S.: Solutions of he nonlinear integral equation and fractional differential equation using the technique of a fixed point with a numerical experiment in extended $b$-metric space. Symmetry 11, 686 (2019)

17. Adiguzel, R.S., Aksoy, U., Karapinar, E., Erhan, I.M.: On the solution of a boundary value problem associated with a fractional differential equation. Math. Methods Appl. Sci. (2020). https://doi.org/10.1002/mma.6652

18. Alqahtani, B., Aydi, H., Karapinar, E., Rakocevic, V.: A solution for Volterra fractional integral equations by hybrid contractions. Mathematics 7, 694 (2019)

19. Karapınar, E., Fulga, A., Rashid, M., Shahid, L., Aydi, H.: Large contractions on quasi-metric spaces with an application to nonlinear fractional differential-equations. Mathematics 7, 444 (2019)

20. Karapinar, E., Abdeljawad, T., Jarad, F.: Applying new fixed point theorems on fractional and ordinary differential equations. Adv. Differ. Equ. 2019, 421 (2019)

21. Alqahtania, B., Fulga, A., Jarad, F., Karapinar, E.: Nonlinear F-contractions on b-metric spaces and differential equations in the frame of fractional derivatives with Mittag-Leffler kernel. Chaos Solitons Fractals 128, 349-354 (2019)

22. Ravichandrana, C., Logeswaria, K., Jarad, F.: New results on existence in the framework of Atangana-Baleanu derivative for fractional integro-differential equations. Chaos Solitons Fractals 125, 194-200 (2019)

23. Shoaib, M., Abdeljawad, T., Sarwar, M., Jarad, F.: Fixed point theorems for multi-valued contractions in $b$-metric spaces with applications to fractional differential and integral equations. IEEE Access 7, 127373-127383 (2019)

24. Kanwal, T., Hussain, A., Baghani, H., de la Sen, M.: New fixed point theorems in orthogonal F-metric spaces with application to fractional differential equation. Symmetry 12, 832 (2020)

25. Hussain, A.: Fractional convex type contraction with solution of fractional differential equation. AIMS Math. 5(5), 5364-5380 (2020)

26. Karapinar, E.: Revisiting the Kannan type contractions via interpolation. Adv. Theory Nonlinear Anal. Appl. 2, 85-87 (2018). https://doi.org/10.31197/atnaa.431135

27. Karapinar, E., Agarwal, R.P., Aydi, H.: Interpolative Reich-Rus-Ciric type contractions on partial metric spaces. Mathematics 6, 256 (2018). https://doi.org/10.3390/math6110256

28. Aydi, H., Karapinar, E., Roldán López de Hierro, A.F.: $\omega$-Interpolative Ciric-Reich-Rus-type contractions. Mathematics 7 57 (2019)

29. Aydi, H., Chen, C.-M., Karapinar, E.: Interpolative Ciric-Reich-Rus type contractions via the Branciari distance. Mathematics 7(1), 84 (2019). https://doi.org/10.3390/math7010084

30. Karapinar, E., Alqahtani, O., Aydi, H.: On interpolative Hardy-Rogers-type contractions. Symmetry 11(1), 8 (2019). https://doi.org/10.3390/sym11010008

31. Gaba, Y.U., Karapinar, E.: A new approach to the interpolative contractions. Axioms 8, 110 (2019)

32. Agarwal, R.P., Karapinar, E.: Interpolative Rus-Reich-Ciric-type contractions via simulation functions. An. Ştiinţ. Univ. 'Ovidius' Constanţa, Ser. Mat. 27(3), 137-152 (2019)

33. Samet, B., Vetro, C., Vetro, P.: Fixed point theorems for $\alpha-\psi$-contractive-type mappings. Nonlinear Anal. 75, 2154-2165 (2012)

34. Salimi, P., Latif, A., Hussain, N.: Modified $\alpha-\psi$-contractive mappings with applications. Fixed Point Theory Appl. 2013 $151(2013)$

35. Hussain, N., Salimi, P.: Suzuki-Wardowski type fixed point theorems for $\alpha$-GF-contractions. Taiwan. J. Math. 18(6), 1879-1895 (2014). https://doi.org/10.11650/tjm.18.2014.4462

36. Karapinar, E., Samet, B.: Generalized $(\alpha-\psi)$-contractive type mappings and related fixed point theorems with applications. Abstr. Appl. Anal. 2012, Article ID 793486 (2012) 
37. Kutbi, M.A., Arshad, M., Hussain, A.: On modified $\alpha-\eta$-contractive mappings. Abstr. Appl. Anal., 2014, Article ID 657858 (2014)

38. Hussain, N., Latif, A., Iqbal, I.: Fixed point results for generalized F-contractions in modular metric and fuzzy metric spaces. Fixed Point Theory Appl. 2015, 158 (2015)

Submit your manuscript to a SpringerOpen ${ }^{\circ}$ journal and benefit from:

- Convenient online submission

- Rigorous peer review

- Open access: articles freely available online

- High visibility within the field

- Retaining the copyright to your article

Submit your next manuscript at $\gg$ springeropen.com 